(C) 2015 IEEE. Personal use of this material is permitted. Permission from IEEE must be obtained for all other uses, in any current or future media, including reprinting/republishing this material for advertising or promotional purposes, creating new collective works, for resale or redistribution to servers or lists, or reuse of any copyrighted component of this work in other works.

IEEE Transactions on computers, epub ahead of print 04 March 2015

D.O.I: $\underline{10.1109 / T C .2015 .2409849}$ 


\title{
Using Flexibility in P-Circuits by Boolean Relations
}

\author{
Anna Bernasconi, Valentina Ciriani, Gabriella Trucco, and Tiziano Villa
}

\begin{abstract}
In this paper we study the problem of characterizing and exploiting the complete flexibility of a special logic architecture, called P-circuits, which realize a Boolean function by projecting it onto overlapping subsets given by a generalized Shannon decomposition. P-circuits are used to restructure logic by pushing some signals towards the outputs. The algorithms proposed so far for exploiting the structural flexibility of P-circuits do not guarantee to find the best implementation, because they cast the problem as the minimization of an incompletely specified function. Instead, here we show that to explore all solutions we must set up the problem as the minimization of a Boolean relation, because there are don't care conditions that cannot be expressed by single cubes. Finally we report the results obtained using a minimizer of Boolean relations, which improve in a major way with respect to the previously literature.
\end{abstract}

Index Terms-Logic synthesis, Boolean decomposition, Boolean relations

\section{INTRODUCTION}

Logic optimization of digital circuits explores different realizations of a logic circuit to improve design parameters like area, speed, power consumption, and others. This goal is achieved exploiting both the flexibility allowed by the specification (external don't care conditions), and the internal or structural flexibility due to the structure of the implementation. Therefore, given a chosen logic architecture, the goal of logic synthesis is from one side to characterize completely the implementations consistent with the specification and the architecture, and from other side to explore all of them choosing the best with respect to a given cost function.

For instance, in the model of multi-level synthesis $a$ la SIS [13], based on Boolean networks whose nodes are single-output PLAs, two sources of flexibility were defined to characterize the complete flexibility: satisfiability don't cares, SDCs, to model limited controllability, and observability don't cares, ODCs, to model limited observability. Then, algorithms were proposed to compute part or all of them, and the flexibility was exploited running two-level minimization on the single nodes of the Boolean network.

In this paper we revisit the problem of characterizing and exploiting the complete flexibility of a special logic architecture, called P-circuits. They are extended forms of Shannon cofactoring, investigated in [3], [4], [6], where the expansion is with respect to an orthogonal basis $\bar{x}_{i} \oplus p$ (i.e.,

- A. Bernasconi is with the Department of Computer Science, Università di Pisa, Italy.

E-mail: anna.bernasconi@unipi.it

- V. Ciriani and G. Trucco are with the Department of Computer Science, Università degli Studi di Milano, Italy. \{valentina.ciriani, gabriella.trucco\}@unimi.it

- T. Villa is with the Department of Computer Science, Università degli Studi di Verona, Italy.

tiziano.villa@univrit $x_{i}=p$ ), and $x_{i} \oplus p$ (i.e., $x_{i} \neq p$ ), where $p$ is a function defined over all variables except for a critical variable $x_{i}$. P-circuits were introduced as a specialized form of decomposition with respect to specific critical signals that should be pushed closer to the outputs, e.g., signals with the highest switching activity (to decrease power consumption), or late-arriving ones (to decrease worst-case delay).

More in detail, let $f_{x_{i}=p}$ and $f_{x_{i} \neq p}$ be the projections of a function $f$ onto $x_{i}=p$ and $x_{i} \neq p$, and let $I=$ $f_{x_{i}=p} \cap f_{x_{i} \neq p}$ be the points common to the two projections. A completely defined function $f$ can be decomposed giving three Boolean functions combined by a disjunction: $f=$ $\left(\bar{x}_{i} \oplus p\right) f^{=}+\left(x_{i} \oplus p\right) f^{\neq}+f^{I}$, where $f^{=\subseteq f_{x_{i}=p}, f^{\neq} \subseteq}$ $f_{x_{i} \neq p}$, and $f^{I} \subseteq I$. The circuits synthesized according to this structure are called P-circuits when the blocks $f=$, $f^{\neq}$and $f^{I}$ are realized by sums-of-products (as shown in Figure 1). Shannon decomposition is the special case when $p=0, f^{=}=f_{x_{i}=p}, f^{\neq}=f_{x_{i} \neq p}$, and $f^{I}=\emptyset$.

The structural flexibility arises in P-circuits as follows: if a point $q$ of $f$ is covered in one set $\left(f^{=}, f^{\neq}\right.$, or $\left.f^{I}\right)$, $q$ may be considered as a don't care in the other sets containing it. This can be seen as a special case of the following disjunctive paradigm: when the outputs of some Boolean functions are combined by a disjunction, if a single function produces the output value 1 for some inputs, we do not care about the output values produced by the remaining functions for such inputs; therefore under these inputs the representation of the other functions can be modified and optimized according to a chosen cost function [14].

The computation of the flexibility of P-circuits has been already studied in the literature [3], [4], [6], but the algorithms proposed so far for its exploitation may fail to find the best implementation, because they cast the optimization problem as the minimization of an incompletely specified function. Instead, in this paper we show that to explore all solutions we must set up the problem as the minimization of a Boolean relation, because there are combinations of 


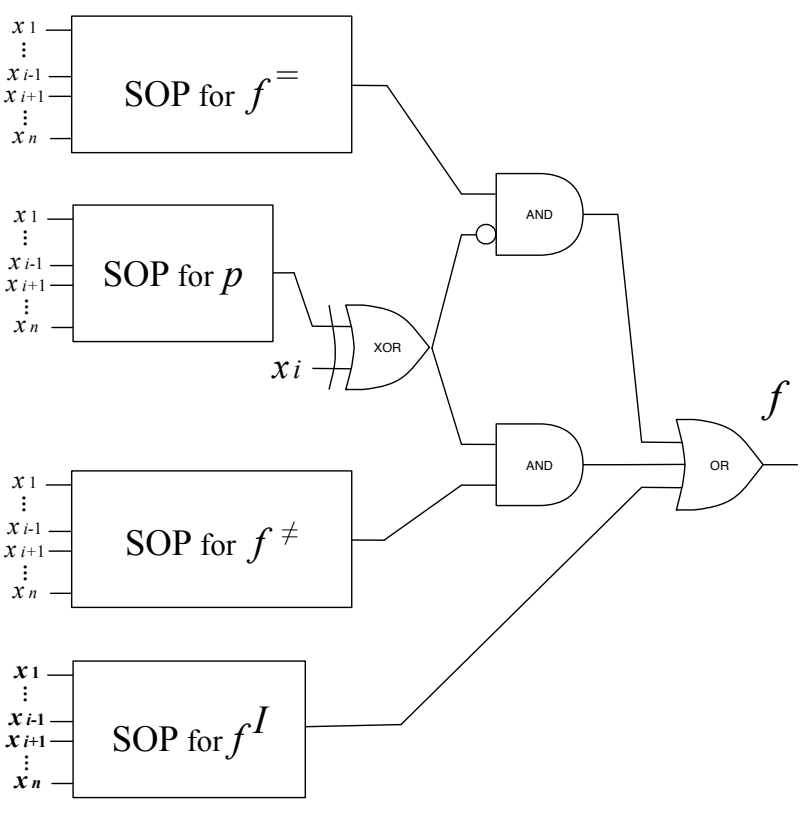

Fig. 1. A P-circuit for the function $f$ decomposed with respect to the critical variable $x_{i}$ and the function $p$.

don't care conditions that cannot be expressed by single cubes. Therefore here we model the optimal P-circuit decomposition problem by means of Boolean relations, and show that it is a complete characterization of the structural flexibility of P-circuits. Finally we report the results obtained using a minimizer of Boolean relations, which are a major improvement with respect to the previously published experiments.

This paper is an extended version of the conference paper presented in [5] and is organized as follows. Section 2 introduces P-circuits for completely and incompletely specified functions. Section 3 describes how to minimize P-circuits using Boolean relations. Experiments on a set of benchmarks are reported in Section 4, and Section 5 concludes the paper.

\section{P-CIRCUITS}

A P-circuit is a network where the dependence on a given variable $x_{i}$ (e.g., the variable with more switching activity or with higher delay) is projected away from the rest of the circuit.

In this section we first briefly review the P-circuits based on SOP minimization [3], [4], [6]. We then give a new formulation of the associated minimization problem, both for completely and incompletely specified functions, better suited to be formalized via Boolean relations.

We first give some preliminary definitions. A completely specified Boolean function $f$ is a function $f:\{0,1\}^{n} \rightarrow$ $\{0,1\}$. A completely specified Boolean function can also be interpreted as the set of points $x \in\{0,1\}^{n}$ such that $f(x)=$ 1. An incompletely specified Boolean function is a function $f:\{0,1\}^{n} \rightarrow\{0,1,-\}$, where - is called the don't care value of the function. An incompletely specified function

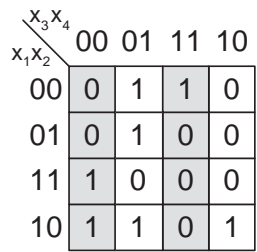

(a) $f$

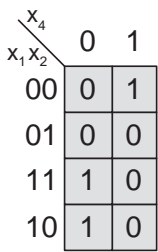

(b) $\left.\mathrm{f}\right|_{\mathrm{x} 3=\mathrm{x} 4}$
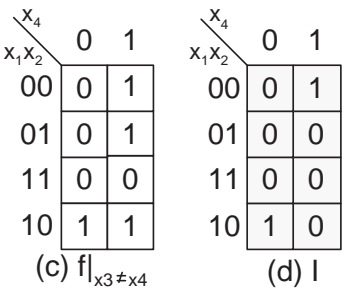

Fig. 2. A function $f(\mathrm{a})$, the corresponding projected functions $\left.f\right|_{x_{3}=x_{4}}$ and $\left.f\right|_{x_{3} \neq x_{4}}$ (b) and (c), and their intersection I (d).

can be described by three sets of points: the on-set, the off-set and the don't care set, which characterize the points in $\{0,1\}^{n}$ with images 0,1 , and - , respectively. Given the Boolean space $\{0,1\}^{n}$ described by the set $\left\{x_{1}, \ldots, x_{n}\right\}$ of $n$ binary variables, a literal is a variable or its complement; a cube is conjunction (or product) of a set of literals, and a minterm is a cube when it represents only one point, i.e., when it is a conjunction of $n$ literals. Finally, a multipleoutput Boolean function $f$ is a function $f:\{0,1\}^{n} \rightarrow$ $\{0,1,-\}^{m}$; it can be considered also as a vector of Boolean functions $\left\{f_{1}, f_{2}, \ldots, f_{m}\right\}$.

\subsection{Completely Specified Functions}

Let $f$ be a completely specified Boolean function depending on the set $\left\{x_{1}, \ldots, x_{n}\right\}$ of $n$ binary variables. The classical Shannon decomposition $f=\left.\bar{x}_{i} f\right|_{\bar{x}_{i}}+\left.x_{i} f\right|_{x_{i}}$, and the more general EXOR-based decomposition [7] [11]

$$
f=\left.\left(\bar{x}_{i} \oplus p\right) f\right|_{x_{i}=p}+\left.\left(x_{i} \oplus p\right) f\right|_{x_{i} \neq p}
$$

(where $p$ is a function non-depending on $\left.x_{i}\right)^{1}$ are suitable for keeping $x_{i}$ disjoint from the rest of the circuit, but are not oriented to area minimization. In fact, $\left.f\right|_{x_{i}=p}$, and $\left.f\right|_{x_{i} \neq p}$ do not depend on the variable $x_{i}$, but the cubes of $f$ intersecting both subsets $x_{i}=p$ and $x_{i} \neq p$ may be split into two smaller subcubes when they are projected onto $\left.f\right|_{x_{i}=p}$, and $\left.f\right|_{x_{i} \neq p}$, respectively. For example, consider the function $f$ in Figure 2 (a), the variable $x_{i}=x_{3}$ and the simple function $p\left(x_{1}, x_{2}, x_{4}\right)=x_{4}$. The projection of $f$ with respect to $x_{3}=x_{4}$ and $x_{3} \neq x_{4}$ are depicted in Figures 2 (b) and (c), respectively. We note that the cube $\bar{x}_{1} \bar{x}_{2} x_{4}$ of $f$ is split into two separate minterms $\bar{x}_{1} \bar{x}_{2} x_{4}$ and $\bar{x}_{1} \bar{x}_{2} x_{4}$ in the two projected functions $\left.f\right|_{x_{3}=x_{4}}$ and $\left.f\right|_{x_{3} \neq x_{4}}$ (Figures 2 (b) and (c)).

To overcome this problem, in P-circuits synthesis, we keep unprojected some of the points of the original function. For this purpose, let $I=\left.\left.f\right|_{x_{i}=p} \cap f\right|_{x_{i} \neq p}$ be the intersection of the two cofactors $\left.f\right|_{x_{i}=p}$ and $\left.f\right|_{x_{i} \neq p}$. Note that the intersection $I$ contains the cubes whose products do not contain $x_{i}$ and that cross the two sets. Therefore, in order to overcome the splitting of these crossing cubes, we could keep $I$ unprojected, and project only the minterms in

1. Note that the Shannon decomposition is a particular EXOR-based decomposition where $\mathrm{p}=0$. 


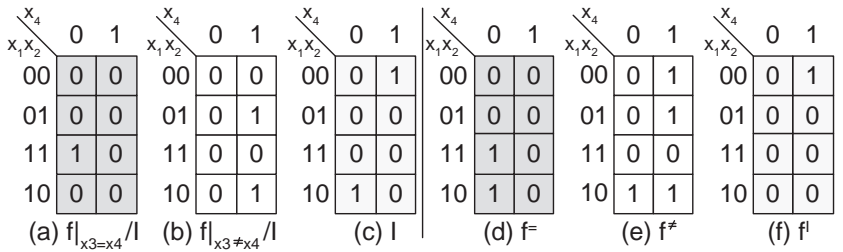

Fig. 3. Running example.

$\left.f\right|_{x_{i}=p} \backslash I$ and $\left.f\right|_{x_{i} \neq p} \backslash I$, obtaining the expression

$$
f=\left(\bar{x}_{i} \oplus p\right)\left(\left.f\right|_{x_{i}=p} \backslash I\right)+\left(x_{i} \oplus p\right)\left(\left.f\right|_{x_{i} \neq p} \backslash I\right)+I .
$$

Note that $p,\left.f\right|_{x_{i}=p} \backslash I,\left.f\right|_{x_{i} \neq p} \backslash I$ and $I$ do not depend on $x_{i}$. Following the previous example, see Figures 3 (a), (b), and (c).

However, the points that are in $I$ could be exploited to form bigger cubes in the projected sets. For example, the point 001 of $I$ would be useful for forming the cube $\bar{x}_{1} x_{4}$ in $\left.f\right|_{x_{3} \neq x_{4}} \backslash I$, see Figure 3 (b), indeed such cube was originally in $\left.f\right|_{x_{3} \neq x_{4}}$, see Figure 2 (c). Therefore, if a point is in $I$ and is useful for a better minimization of the projected parts, it can be kept both in the projection and in the intersection (see, for example, the point 001 in Figures 3 (e) and (f)). Moreover, if a point is covered in both the projected sets, it is not necessary to cover it in the intersection. In our example, the point 100 of $I$ is used for forming bigger cubes in both the projections (Figures 3 (d) and (e)), thus it can be removed from the intersection (see Figure 3 (f)).

In the following, we indicate a SOP circuit implementing a Boolean function $f$ with $S(f)$, and we denote optimal circuits (in the sense of two-level minimization) with a star, as $S^{*}(f)$.

From the previous observations, we can infer that the projected sub-circuits should cover at least $\left.f\right|_{x_{i}=p} \backslash I$ and $\left.f\right|_{x_{i} \neq p} \backslash I$, and must be contained in $\left.f\right|_{x_{i}=p}$ and $\left.f\right|_{x_{i} \neq p}$, respectively. Moreover, the part of the circuit that is not projected should be contained in the intersection $I$.

In summary, we rephrase the definition of a P-circuit given in [6] as follows.

Definition 1: A P-circuit of a completely specified function $f$ is the circuit $P(f)$ denoted by the expression:

$P(f)=\left(\bar{x}_{i} \oplus S(p)\right) S\left(f^{=}\right)+\left(x_{i} \oplus S(p)\right) S\left(f^{\neq}\right)+S\left(f^{I}\right)$

where

1) $\left.\left(\left.f\right|_{x_{i}=p} \backslash I\right) \subseteq f^{=} \subseteq f\right|_{x_{i}=p}$

2) $\left.\left(\left.f\right|_{x_{i} \neq p} \backslash I\right) \subseteq f^{\neq} \subseteq f\right|_{x_{i} \neq p}$

3) $\emptyset \subseteq f^{I} \subseteq I$

4) $P(f)=f$.

Therefore, the synthesis idea of P-circuits is to construct a network for $f$ by appropriately choosing the sets $f^{=}, f^{\neq}$, and $f^{I}$ as building blocks (as shown in Figure 1).

The cofactors and the intersection can be synthesized in any framework of logic minimization. Here we focus on the standard SOP synthesis, since SOP forms are widely used and also because we exploit the Boolean relation minimizer
BREL [2] that is built for classical SOP synthesis. As far as we know, no Boolean relation minimizer is available for other logic forms. Thus, we represent $f^{=}, f^{\neq}$, and $f^{I}$ as sums of products.

For example, the expression

$$
P(f)=\left(x_{3} \oplus x_{4}\right)\left(x_{1} \bar{x}_{2}+\bar{x}_{1} x_{4}\right)+\left(\bar{x}_{3} \oplus x_{4}\right)\left(x_{1} \bar{x}_{4}\right)+\bar{x}_{1} \bar{x}_{2} x_{4}
$$

is a P-circuit for the function $f$ defined in Figure 2 (a). $P(f)$ is derived from the functions $f^{=}, f^{\neq}$, and $f^{I}$ in Figures 3 (d), (e), and (f).

An optimal P-circuit $P^{*}(f)$ for the function $f$ is a $\mathrm{P}$ circuit with minimum cost that can be synthesized for $f$. The P-circuit described in the previous example is an optimal P-circuit with respect to the number of literals in the SOP forms.

\subsection{Incompletely Specified Functions}

Consider now an incompletely specified Boolean function $f=\left\{f^{o n}, f^{d c}\right\}$. For the sake of simplicity, suppose that $f^{o n} \cap f^{d c}=\emptyset$; otherwise, following the usual semantics, we consider $f^{o n} \backslash f^{d c}$ as the on-set of $f$. Let $I$ be the intersection of the projections of $f$ onto the two sets $x_{i}=p$ and $x_{i} \neq p$ :

$$
I=\left(\left.\left.f^{o n}\right|_{x_{i}=p} \cup f^{d c}\right|_{x_{i}=p}\right) \cap\left(\left.\left.f^{o n}\right|_{x_{i} \neq p} \cup f^{d c}\right|_{x_{i} \neq p}\right) .
$$

We can generalize the definition of P-circuit in the following way.

Definition 2: A P-circuit of an incompletely specified function $f=\left\{f^{o n}, f^{d c}\right\}$ is the circuit $P(f)$ denoted by the expression:

$P(f)=\left(\bar{x}_{i} \oplus S(p)\right) S\left(f^{=}\right)+\left(x_{i} \oplus S(p)\right) S\left(f^{\neq}\right)+S\left(f^{I}\right)$

where
1) $\left.\left.\left(\left.f^{o n}\right|_{x_{i}=p} \backslash I\right) \subseteq f^{=} \subseteq f^{o n}\right|_{x_{i}=p} \cup f^{d c}\right|_{x_{i}=p}$
2) $\left.\left.\left(\left.f^{o n}\right|_{x_{i} \neq p} \backslash I\right) \subseteq f^{\neq} \subseteq f^{o n}\right|_{x_{i} \neq p} \cup f^{d c}\right|_{x_{i} \neq p}$
3) $\emptyset \subseteq f^{I} \subseteq I$
4) $f^{o n} \subseteq P(f) \subseteq f^{o n} \cup f^{d c}$.

\section{Minimization of P-Circuits USING Boolean RELATIONS}

The problem of minimizing a Boolean function in P-circuit form can be characterized completely by using Boolean relations, as explained in the following subsections, first for completely specified and then for incompletely specified single-output functions. We will then discuss some optimization issues and how to deal with multi-output functions.

\subsection{Boolean Relations}

In this subsection we recall the theory of Boolean relations, and give a brief overview of the recursive paradigm to solve Boolean relations presented in [10], [1], [2].

The concept of Boolean relations was introduced in [8] as a more general scheme for the incomplete specification of logic networks. In fact, the conditions under which a 


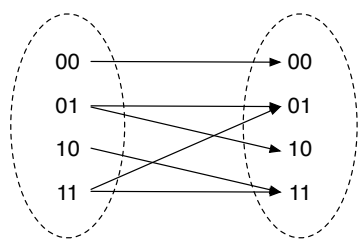

\begin{tabular}{|c|c|}
\hline$x_{1} x_{2}$ & $y_{1} y_{2}$ \\
\hline 00 & $\{00\}$ \\
01 & $\{01,10\}$ \\
10 & $\{11\}$ \\
11 & $\{01,11\}$ \\
\hline
\end{tabular}

Fig. 4. An example of a Boolean relation in graphical (left) and tabular (right) form.

multi-output logic network can be simplified cannot always be completely represented using don't cares.

Definition 3 ([8]): A Boolean relation is a one-to-many multi-output Boolean mapping $\mathcal{R}:\{0,1\}^{n} \rightarrow\{0,1\}^{m}$. $\{0,1\}^{n}$ and $\{0,1\}^{m}$ are called the input and output sets of $\mathcal{R}$.

A Boolean relation $\mathcal{R}$ can be considered a generalization of a Boolean function, where a point in the input set $\{0,1\}^{n}$ can be associated with several points in the output set $\{0,1\}^{m}$; indeed, because of the one-to-many nature of Boolean relations, there may be several equivalent outputs for a given input. Also, notice that $\mathcal{R}$ is a subset of the Cartesian product $\{0,1\}^{n} \times\{0,1\}^{m}$.

A relation $\mathcal{R}$ is well-defined if for all $x \in\{0,1\}^{n}$, there is $y \in\{0,1\}^{m}$ such that $(x, y) \in \mathcal{R}$. A well defined Boolean relation is functional if every input $x$ is associated with one and only one output $y$.

Figure 4 shows an example of a Boolean relation with two input and two output variables, in tabular and in graphical representation. This relation is not a functional one, indeed the inputs 01 and 11 are associated to two outputs each: $\{01,10\}$ and $\{01,11\}$ respectively. Also observe that while the outputs associated to 11 could be expressed by the single output $\{-1\}$ introducing a don't care into the range of output variables, the two outputs related to 01 cannot be expressed using don't cares. As even this simple example shows, Boolean relations capture more flexibility than incompletely specified Boolean functions.

To any relation $\mathcal{R}$ we can associate a set of compatible multi-output Boolean functions, i.e. the set of all functions $f$ such that, for all input $x \in\{0,1\}^{n}, f(x)$ is contained in the set $\mathcal{R}(x)$ of the outputs related to $x$. In this case, we write $f \subseteq \mathcal{R}$.

Definition 4 ([2]): The set of multi-output Boolean functions compatible with a Boolean relation $\mathcal{R}$ : $\{0,1\}^{n} \rightarrow\{0,1\}^{m}$, is defined as $\mathcal{F}(\mathcal{R})=\left\{f:\{0,1\}^{n} \rightarrow\right.$ $\{0,1\}^{m} \mid f \subseteq \mathcal{R}$ and $f$ is a function $\}$.

Two Boolean functions compatible with the Boolean relation of Figure 4 are shown in Figure 5.

The problem of the optimal implementation of a Boolean relation $\mathcal{R}$ is the one of selecting, among the possible functions compatible with $\mathcal{R}$, one of minimum cost according to a given metric. More precisely

Definition 5 ([2]): The solution of a Boolean relation $\mathcal{R}$ is a multi-output Boolean function $f \in \mathcal{F}(\mathcal{R})$. The function $f$ is an optimal solution of $\mathcal{R}$ according to a given cost function $c$, if for all $f^{\prime} \in \mathcal{F}(\mathcal{R}), c(f) \leq c\left(f^{\prime}\right)$.

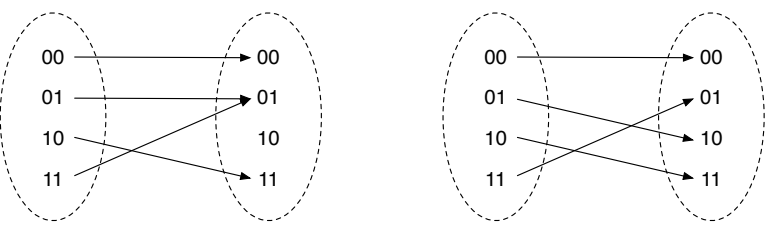

Fig. 5. Two Boolean functions compatible with the relation of Figure 4.

Many problems in logic synthesis can be formalized using Boolean relations, and Boolean decomposition is one of them. For instance, consider the decomposition of a Boolean function $f:\{0,1\}^{n} \rightarrow\{0,1\}$ using a function $g:\{0,1\}^{m} \rightarrow\{0,1\}:$

$f\left(x_{1}, \ldots, x_{n}\right)=g\left(f_{1}\left(\left(x_{1}, \ldots, x_{n}\right), \ldots, f_{m}\left(x_{1}, \ldots, x_{n}\right)\right)\right.$.

The relation $\mathcal{R}$ that represents all possible decompositions of $f$ using $g$ can be defined as follows: for all $x \in\{0,1\}^{n}$, $\mathcal{R}(x)=\left\{y \in\{0,1\}^{m} \mid g(y)=f(x)\right\}$. In other words, each input $x \in\{0,1\}^{n}$ where $f(x)=1$, will be associated to the set of outputs $y \in\{0,1\}^{m}$, where $g$ takes the value 1; each input $x \in\{0,1\}^{n}$ where $f(x)=0$, will be associated to the set of outputs $y \in\{0,1\}^{m}$, where $g$ is 0 . A solver of Boolean relations will explore the set of possible solutions of the relation $\mathcal{R}$ and will return an optimal solution according to the minimization objective.

Several exact and heuristic algorithms have been proposed for solving Boolean relations (see [2] for an overview of these methods, and for bibliographic references). For our particular minimization problem, we used the recursive algorithm proposed in [2] because of its ability in finding better solutions in shorter runtime than the previously known methods. In fact, this recursive solver is very efficient and able to explore a wide space of solutions. Moreover it can be used both in an exact and heuristic approximate mode and its cost function can be tuned for different parameters related to the area or the delay in computing the relation.

The idea of the recursive approach is that of reducing the problem of solving a Boolean relation to a sequence of Boolean minimization problems applied to multi-output incompletely specified functions. Each of these functions is an overapproximation of the original Boolean relation, obtained by expanding the inputs whose outputs cannot be expressed using don't cares to cover more points of the output set. For example, the relation of Figure 4 can be overapproximated by the function

\begin{tabular}{|c|c|}
\hline$x_{1} x_{2}$ & $y_{1} y_{2}$ \\
\hline 00 & 00 \\
01 & -- \\
10 & 11 \\
11 & -1 \\
\hline
\end{tabular}

obtained by expanding the set $\mathcal{R}(01)=\{01,10\}$ of the outputs related to 01 to $\{00,01,10,11\}=--$. 
The function that overapproximates the relation is then minimized using a standard minimization method. If the minimized function is compatible with the relation, the algorithm reports the results. Otherwise, if the function contains conflicts, the algorithm continues with a successive refinement of the original relation into two smaller relations containing fewer outputs that cannot be expressed using don't cares. These smaller relations, obtained by selecting an input where there is an incompatibility and partitioning its outputs, are solved recursively, and the best compatible solution is selected out of the explored solutions. For instance, the minimal SOP cover of the function $f$ that overapproximates the relation $\mathcal{R}$ of Figure 4 would be (in PLA form):

$$
\begin{array}{ll}
0- & 00 \\
1- & 11
\end{array}
$$

corresponding to a function not compatible with $\mathcal{R}$, as $f(01)=00$ but $00 \notin \mathcal{R}(01)$. Thus, the algorithm splits $\mathcal{R}$ into the two smaller relations

\begin{tabular}{|c|c|}
\hline$x_{1} x_{2}$ & $y_{1} y_{2}$ \\
\hline 00 & 00 \\
01 & 01 \\
10 & 11 \\
11 & -1 \\
\hline
\end{tabular}

\begin{tabular}{|c|c|}
\hline$x_{1} x_{2}$ & $y_{1} y_{2}$ \\
\hline 00 & 00 \\
01 & 10 \\
10 & 11 \\
11 & -1 \\
\hline
\end{tabular}

both corresponding to incompletely specified Boolean functions. Once the two functions have been minimized, the best cover is selected and reported as solution of the original relation $\mathcal{R}$.

\subsection{Completely Specified Functions}

Let $f$ be a completely specified Boolean function depending on $n$ variables, $x_{i}$ one input variable, and $p$ a function depending on all input variables of $f$ but $x_{i}$. Consider the two cofactors $\left.f\right|_{x_{i}=p}$ and $\left.f\right|_{x_{i} \neq p}$, obtained by projecting $f$ onto the sets $x_{i}=p$ and $x_{i} \neq p$, and their intersection $I=\left.\left.f\right|_{x_{i}=p} \cap f\right|_{x_{i} \neq p}$.

As recalled in Section 2, in order to minimize $f$ in P-circuit form, we must find a refinement of the starting partition of the minterms of $f$ into the three disjoint sets $\left.f\right|_{x_{i}=p} \backslash I,\left.f\right|_{x_{i} \neq p} \backslash I$ and $I$. This refinement, which leads to the three new sets $f^{=}, f^{\neq}$, and $f^{I}$, consists in $(i)$ adding back some points of $I$ to $\left.f\right|_{x_{i}=p} \backslash I$ or $\left.f\right|_{x_{i} \neq p} \backslash I$, precisely the points that may help in obtaining larger cubes in their SOP representation, and (ii) subtracting from $I$ some of the points in the intersection of the refined cofactors (i.e., covered by both cofactors), in order to find a more compact SOP representation for the intersection set.

The final P-circuit for $f$ is then given by three minimal SOPs representing $f^{=}, f^{\neq}$, and $f^{I}$, appropriately connected to a minimal SOP for $p$. Therefore, the problem is finding the sets $\left(f^{=}, f^{\neq}, f^{I}\right)$ that lead to a P-circuit of minimal cost, according to a given cost metric. We now show how this problem can be nicely formalized and efficiently solved using Boolean relations.
Our aim is to define a relation $\mathcal{R}$ whose set of compatible functions $\mathcal{F}(\mathcal{R})$ corresponds exactly to the set of all possible P-circuits for $f$, so that an optimal solution of $\mathcal{R}$ is an optimal P-circuit $P^{*}(f)$ for $f$.

Let $\mathcal{R}_{f}:\{0,1\}^{n-1} \rightarrow\{0,1\}^{3}$ be a Boolean relation, whose input set is the space spanned by the variables $x_{1} \ldots x_{i-1} x_{i+1} \ldots x_{n}$, while the output set describes all possible tuples of functions $f^{=}, f^{\neq}, f^{I}$ defining a P-circuit for $f$.

From Definition 1, we can observe that the sets $\left.f\right|_{x_{i}=p} \backslash I$ and $\left.f\right|_{x_{i} \neq p} \backslash I$ must be always contained in $f^{=}$and $f^{\neq}$, respectively. Thus, all points in $\left.f\right|_{x_{i}=p} \backslash I$ are associated to the output 100, and all points in $\left.f\right|_{x_{i} \neq p} \backslash I$ are associated to the output 010 of $\mathcal{R}_{f}$.

Moreover, we can also note that the possible P-circuit implementations of $f$ differ in the distribution of the points of $I$ among the three sets $f^{=}, f^{\neq}, f^{I}$. In particular, each minterm in $I$ can be associated to one of the following outputs of $\mathcal{R}_{f}$ :

- 001 , if it belongs only to $f^{I}$, that is, it has been kept in $I$, and not added to $f^{=}$and $f^{\neq}$;

- 101 , if it has been added to $f^{=}$and kept in $I$;

- 011, if it has been added to $f^{\neq}$and kept in $I$;

- 111 if it has been added to $f^{=}$and $f^{\neq}$and kept in $I$;

- 110 if it has been added to $f^{=}$and $f^{\neq}$and removed from $I$.

The possible outputs corresponding to the points in $I$ can be represented in a compact way using don't cares: --1 and $11-$. Indeed, if a point is covered in the intersection set, then it becomes a don't care point in the two projection sets $x_{i}=p$ and $x_{i} \neq p$, while if it is covered in both projection sets, it becomes a don't care for $I$.

Since all points of $I$ must be covered either in $f^{I}$, or both in $f^{=}$and $f^{\neq}$, all other possible output choices for $I$ would not define a P-circuit for $f$.

The relation $\mathcal{R}_{f}$ can therefore be defined as follows:

\begin{tabular}{|l|c|}
\hline$x_{1} \ldots x_{i-1} x_{i+1} \ldots x_{n}$ & $\mathcal{R}_{f}$ \\
\hline points in $\left.f\right|_{x_{i}=p} \backslash I$ & $\{100\}$ \\
points in $\left.f\right|_{x_{i} \neq p} \backslash I$ & $\{010\}$ \\
points in $I$ & $\{--1,11-\}$ \\
all other points & $\{000\}$ \\
\hline
\end{tabular}

Observe that $\mathcal{R}_{f}$ is truly a relation, as it is not functional on the points of $I$.

With this formalism, we can rephrase our minimization problem as finding an optimal implementation of $\mathcal{R}_{f}$, that is, as selecting among all possible three-output functions compatible with $\mathcal{R}_{f}$, each corresponding to a tuple $f^{=}$, $f^{\neq}$, and $f^{I}$, the one whose overall SOP representation is minimal. A formal proof of this statement is given in Section 3.4.

The Algorithm in Figure 6 shows the overall synthesis method via Boolean relations. In the algorithm, after the construction of the Boolean relation $R_{f}$, the procedure OPTRelation $\left(R_{f}\right)$ is called. OPTRelation $\left(R_{f}\right)$ finds a minimal solution of the Boolean relation $R_{f}$ with respect 
P-circuit synthesis of completely specified functions via Boolean relations

INPUT: Completely specified function $f, p$, and a variable $x_{i}$.

OUTPUT: A P-circuit network representation for $f$ w.r.t.

$p$ and $x_{i}$.

NOTATION: $f$ is the on-set of the function $f$.

The relation $R_{f}$ is a set of couples $\langle$ input_cube, $\{$ output_cubes $\}\rangle$.

$R_{f}=\emptyset$;

$I=\left.\left.f\right|_{x_{i}=p} \cap f\right|_{x_{i} \neq p}$;

for each cube $c \in I$

$R_{f}=R_{f} \cup\langle c,\{--1,11-\}\rangle ;$

$f^{(=)}=\left.f\right|_{x_{i}=p} \backslash I$;

for each cube $c \in f(=)$

$R_{f}=R_{f} \cup\langle c,\{100\}\rangle$;

$f^{(\neq)}=\left.f\right|_{x_{i} \neq p} \backslash I$;

for each cube $c \in f(\neq)$

$R_{f}=R_{f} \cup\langle c,\{010\}\rangle ;$

$f_{\text {off }}=\{0,1\}^{n-1} \backslash\left(\left.\left.f\right|_{x_{i}=p} \cup f\right|_{x_{i} \neq p}\right)$;

for each cube $c \in f_{o f f}$

$R_{f}=R_{f} \cup\langle c,\{000\}\rangle ;$

$\left(f_{o p t}^{=}, f_{o p t}^{\neq}, f_{o p t}^{I}\right)=O P T R e l a t i o n ~\left(R_{f}\right)$;

return $\left(\bar{x}_{i} \oplus p\right) f_{o p t}^{=}+\left(x_{i} \oplus p\right) f_{o p t}^{\neq}+f_{o p t}^{I} ;$

Fig. 6. Algorithm for the synthesis of P-circuits of completely specified functions.

to the number of literals in the SOP forms for $f_{=}, f_{\neq}$, and $f_{I}$. The solutions $\left(f_{o p t}^{=}, f_{o p t}^{\neq}, f_{o p t}^{I}\right)$ are given in their minimal SOP form. Thus the Algorithm returns the minimal P-circuit solution.

Example 1: Consider the function in Figure 2, and its projections onto the sets $x_{3}=x_{4}$ and $x_{3} \neq x_{4}$. The intersection between the two cofactors $\left.f\right|_{x_{3}=x_{4}}=$ $\{001,110,100\}$ and $\left.f\right|_{x_{3} \neq x_{4}}=\{001,011,100,101\}$ contains two points $\{001,100\}$. Thus we have

$$
\begin{aligned}
& I=\{001,100\} \\
& \left.f\right|_{x_{3}=x_{4}} \backslash I=\{110\} \\
& \left.f\right|_{x_{3} \neq x_{4}} \backslash I=\{011,101\}
\end{aligned}
$$

The corresponding relation is

\begin{tabular}{|c|c|}
\hline$x_{1} x_{2} x_{4}$ & $\mathcal{R}_{f}$ \\
\hline 001 & $\{11-,--1\}$ \\
011 & $\{010\}$ \\
100 & $\{11-,--1\}$ \\
101 & $\{010\}$ \\
110 & $\{100\}$ \\
\hline
\end{tabular}

where all missing points are associated to the output 000 . It can be easily verified that among all functions compatible with $\mathcal{R}_{f}$, the one whose overall SOP representation is minimal (with respect to the number of literals) is

\begin{tabular}{|c|c|}
\hline$x_{1} x_{2} x_{4}$ & $f^{=} f^{\neq} f^{I}$ \\
\hline 001 & 011 \\
011 & 010 \\
100 & 110 \\
101 & 010 \\
110 & 100 \\
\hline
\end{tabular}

that defines precisely the sets $f=, f^{\neq}$and $f^{I}$ shown in Figures 3 (d), (e), and (f). Thus, the Algorithm in Figure 6 outputs the P-citcuit:

$$
\left(x_{3} \oplus x_{4}\right)\left(x_{1} \bar{x}_{2}+\bar{x}_{1} x_{4}\right)+\left(\bar{x}_{3} \oplus x_{4}\right)\left(x_{1} \bar{x}_{4}\right)+\bar{x}_{1} \bar{x}_{2} x_{4} .
$$

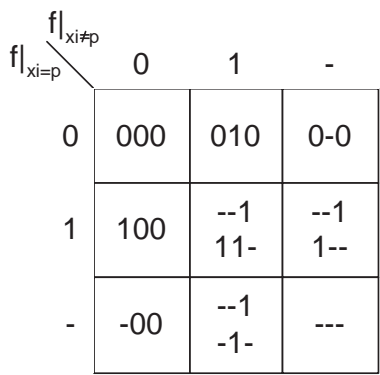

Fig. 7. Outputs for the relation $\mathcal{R}_{f}$ when $f$ is an incompletely specified function.

\section{P-circuit synthesis of incompletely specified functions via Boolean relations}

INPUT: Incompletely specified function $f, p$, and a variable $x_{i}$ OUTPUT: A P-circuit network representation for $f$ w.r.t. $p$ and $x_{i}$.

NOTATION: let $f=\left(f_{o n}, f_{d c}\right)$, i.e., $f_{o n}$ is the on-set of $f$, and $f_{d c}$ is the DC-set of $f$.

The relation $R_{f}$ is a set of couples $\langle$ input_cube, $\{$ output_cubes\}〉.

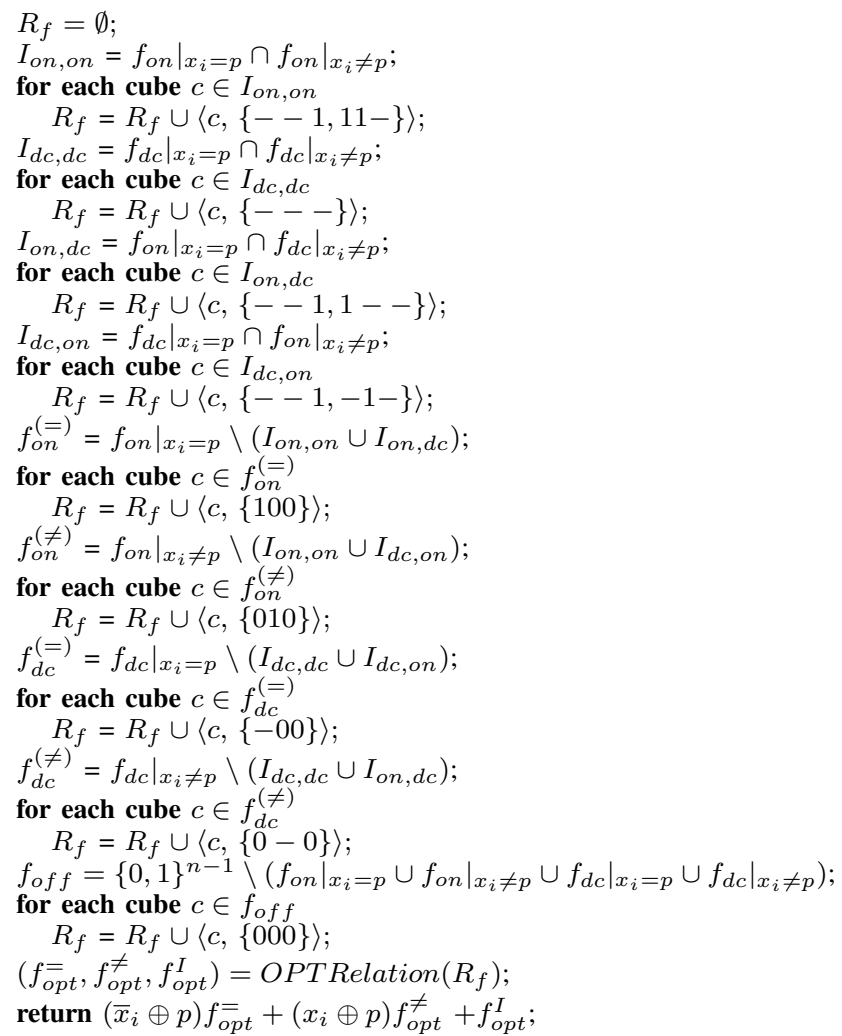

Fig. 8. Algorithm for the synthesis of P-circuits of incompletely specified functions.

\subsection{Incompletely Specified Functions}

Let us now consider the P-circuit minimization of incompletely specified functions. The construction of $\mathcal{R}_{f}$ must consider more cases. In fact, the possible P-circuit implementations depend not only on the distribution of the points of $I$ among the three sets $f=, f^{\neq}, f^{I}$, but also on how the don't care points of the two cofactors are used to build larger cubes in the two projection spaces $x_{i}=p$ and 
$x_{i} \neq p$, and possibly in the intersection $I$.

To correctly define the relation $\mathcal{R}_{f}$ we must partition the input set $\{0,1\}^{n-1}$ into nine subsets, each corresponding to a combination of the values $\{0,1,-\}$ assumed by the two cofactors $\left.f\right|_{x_{i}=p}$ and $\left.f\right|_{x_{i} \neq p}$, as shown in Figure 7. Given $x \in\{0,1\}^{n-1}$, we have the following cases:

1) $\left[\left.f\right|_{x_{i}=p}(x)=0,\left.f\right|_{x_{i} \neq p}(x)=0\right]$ $x$ is a point of both off-sets, thus $\mathcal{R}_{f}(x)=\{000\}$.

2) $\left[\left.f\right|_{x_{i}=p}(x)=0,\left.f\right|_{x_{i} \neq p}(x)=1\right]$ Definition 2 immediately implies that $\mathcal{R}_{f}(x)=$ $\{010\}$.

3) $\left[\left.f\right|_{x_{i}=p}(x)=1,\left.f\right|_{x_{i} \neq p}(x)=0\right]$ As before, Definition 2 implies that $\mathcal{R}_{f}(x)=\{100\}$.

4) $\left[\left.f\right|_{x_{i}=p}(x)=-,\left.f\right|_{x_{i} \neq p}(x)=0\right]$

The don't cares of $\left.f\right|_{x_{i}=p}$, corresponding to zeros of the other cofactor, can be exploited to get a smaller SOP form for $f^{=}$, thus we pose $\mathcal{R}_{f}(x)=\{-00\}$.

5) $\left[\left.f\right|_{x_{i}=p}(x)=0,\left.f\right|_{x_{i} \neq p}(x)=-\right]$

As before, the don't cares of $\left.f\right|_{x_{i} \neq p}$, corresponding to zeros of $\left.f\right|_{x_{i}=p}$, can be exploited to get a smaller SOP form for $f^{\neq}$, thus we pose $\mathcal{R}_{f}(x)=\{0-0\}$.

6) $\left[\left.f\right|_{x_{i}=p}(x)=1,\left.f\right|_{x_{i} \neq p}(x)=1\right]$

The points in the intersection between the on-sets of the two cofactors can be treated exactly as done for completely specified functions, so we have $\mathcal{R}_{f}(x)=$ $\{--1,11-\}$.

7) $\left[\left.f\right|_{x_{i}=p}(x)=1,\left.f\right|_{x_{i} \neq p}(x)=-\right]$

For each point $x$ in the on-set of $\left.f\right|_{x_{i}=p}$ and in the don't care set of $\left.f\right|_{x_{i} \neq p}$ we have two choices: either it is covered in the projection set $x_{i}=p$, thus becoming a don't care point in both the intersection and the other projection set $x_{i} \neq p$, or it can be covered in the intersection and be a don't care in both projection sets. Thus, we pose $\mathcal{R}_{f}(x)=\{1--,--1\}$.

8) $\left[\left.f\right|_{x_{i}=p}(x)=-,\left.f\right|_{x_{i} \neq p}(x)=1\right]$

Analogously, for each point $x$ in don't care set of $\left.f\right|_{x_{i}=p}$ and in the on-set of $\left.f\right|_{x_{i} \neq p}$, we pose $\mathcal{R}_{f}(x)=$ $\{-1-,--1\}$.

9) $\left[\left.f\right|_{x_{i}=p}(x)=-,\left.f\right|_{x_{i} \neq p}(x)=-\right]$

Finally, don't cares of both cofactors can be used as don't cares in all the sets, i.e., $\mathcal{R}_{f}(x)=\{---\}$.

As for completely specified functions, we can rephrase the synthesis of an incompletely specified function $f$ in Pcircuit form as the problem of finding an optimal implementation of $\mathcal{R}_{f}$, i.e., of selecting among all possible threeoutput functions compatible with $\mathcal{R}_{f}$, the one defining a tuple $f^{=}, f^{\neq}$, and $f^{I}$ whose overall SOP representation is minimal (as shown in the Algorithm in Figure 8). A formal proof of this statement is given in the next Section 3.4.

\subsection{Optimality and Complexity Issues}

We now formally prove that the set $\mathcal{F}\left(\mathcal{R}_{f}\right)$ of all threeoutput functions compatible with the relation $\mathcal{R}_{f}$ specifies exactly the set of all tuples $f^{=}, f^{\neq}$, and $f^{I}$ defining a Pcircuit for a Boolean function $f$, so that an optimal solution of $\mathcal{R}_{f}$ is an optimal P-circuit $P^{*}(f)$ for $f$. We consider only the more general problem of minimizing an incompletely specified function in P-circuit form, since it subsumes the problem of minimizing a completely specified function, whose don't care set is just the empty set.

In the following, we first show the results for $p=0$, and then we discuss the case of a general function $p$.

\subsection{1 $\mathrm{p}=0$}

Let $f$ be an incompletely specified Boolean function, $x_{i}$ an input variable, and $R_{f}$ the Boolean relation constructed as explained before. For simplicity consider now the case $p=0$, where $p$ does not need any minimization (note that the experimental results in [3] show that the best choice for the projection function $p$ is the simplest $p=0$ ).

Recall that the three output variables of $R_{f}$ are used to describe the tuple of functions $f^{=}, f^{\neq}$, and $f^{I}$ defining a P-circuit for $f$ : the first two outputs define $f^{=}$and $f^{\neq}$, and the third defines $f^{I}$. Thus, each function in $\mathcal{F}\left(\mathcal{R}_{f}\right)$ corresponds to a possible tuple.

Theorem 1: For $p=0$, the set $\mathcal{F}\left(\mathcal{R}_{f}\right)$ of three-output functions compatible with the relation $\mathcal{R}_{f}$ defines exactly the set of tuples $f^{=}, f^{\neq}$, and $f^{I}$ occurring in all possible P-circuit implementations of $f$.

Proof: First of all observe that any P-circuit $P(f)$ defines a three-output function compatible with $R_{f}$ in an obvious way. The three functions $f^{=}, f^{\neq}$, and $f^{I}$ represented by the three SOPs in $P(f)$ define the three outputs, and we can easily verify that for all $x \in\{0,1\}^{n-1}$, $\left(f^{=}(x), f^{\neq}(x), f^{I}(x)\right) \in \mathcal{R}_{f}(x)$. Indeed, for any $x \in$ $\{0,1\}^{n-1}$

- if $\left(f^{=}(x), f^{\neq}(x), f^{I}(x)\right)=000$, then $\left.f\right|_{x_{i}=p}(x) \in$ $\{0,-\}$ and $\left.f\right|_{x_{i} \neq p}(x) \in\{0,-\}$, and the construction of $\mathcal{R}_{f}$ guarantees that, in each of the possible four cases, $000 \in \mathcal{R}_{f}(x)$ (see Figure 7);

- if $\left(f^{=}(x), f^{\neq}(x), f^{I}(x)\right)=100$, then $\left.f\right|_{x_{i}=p}(x) \in$ $\{1,-\}$ and $\left.f\right|_{x_{i} \neq p}(x) \in\{0,-\}$, and in all cases $100 \in$ $\mathcal{R}_{f}(x)$, by construction (see Figure 7);

- if $\left(f^{=}(x), f^{\neq}(x), f^{I}(x)\right)=010$, then $\left.f\right|_{x_{i}=p}(x) \in$ $\{0,-\}$ and $\left.f\right|_{x_{i} \neq p}(x) \in\{1,-\}$ and in all cases $010 \in$ $\mathcal{R}_{f}(x)$ (see Figure 7);

- if $\left(f^{=}(x), f^{\neq}(x), f^{I}(x)\right) \in\{001,110,101,011,111\}$, then $x \in I$, i.e., $\left.f\right|_{x_{i} \neq p}(x) \in\{1,-\}$ and $\left.f\right|_{x_{i}=p}(x) \in$ $\{1,-\}$, and in all cases the corresponding output $\left(f=(x), f^{\neq}(x), f^{I}(x)\right) \in \mathcal{R}_{f}(x)$ (see Figure 7).

We now prove that any three-output function compatible with $\mathcal{R}_{f}$ defines a P-circuit for $f$.

Consider the first two outputs. The definition of $R_{f}$ (cases (2) and (3)), guarantees that each function in $\mathcal{F}\left(\mathcal{R}_{f}\right)$ assumes value 1 on its first output $f=$ on all points that belong to the on-set of $\left.f\right|_{x_{i}=p}$ and to the off-set of $\left.f\right|_{x_{i} \neq p}$, while the second output $f^{\neq}$is equal to 1 on all points that are in the on-set of $\left.f\right|_{x_{i} \neq p}$ and in the off-set of $\left.f\right|_{x_{i}=p}$. Thus, $f^{=}$contains the subset $\left.f^{o n}\right|_{x_{i}=p} \backslash I$ and $f^{\neq}$contains $\left.f^{o n}\right|_{x_{i} \neq p} \backslash I$, where $I=\left(\left.\left.f^{o n}\right|_{x_{i}=p} \cup f^{d c}\right|_{x_{i}=p}\right) \cap\left(\left.f^{o n}\right|_{x_{i} \neq p} \cup\right.$ $\left.\left.f^{d c}\right|_{x_{i} \neq p}\right)$, as required by Definition 2 .

Now, observe that cases (4) and (5) in the definition of $\mathcal{R}_{f}$ imply that $f^{=}$and $f^{\neq}$could also assume value 1 on all points that belong to the don't care set of the corresponding 
cofactor $\left(\left.f\right|_{x_{i}=p}\right.$ for $f^{=}$and $\left.f\right|_{x_{i} \neq p}$ for $\left.f^{\neq}\right)$and to the offset of the other cofactor.

Furthermore, because of cases (6), (7), (8), and (9) in the definition of $\mathcal{R}_{f}, f^{=}$and $f^{\neq}$might assume value 1 also on the points in the intersection $I$. Finally, note that the relation always sets to 0 the values of $f^{=}$and $f^{\neq}$on all points in the off-set of the corresponding cofactor. Therefore $f=\subseteq$ $\left.\left.f^{o n}\right|_{x_{i}=p} \cup f^{d c}\right|_{x_{i}=p}$ and $\left.\left.f^{\neq} \subseteq f^{o n}\right|_{x_{i} \neq p} \cup f^{d c}\right|_{x_{i} \neq p}$, as required by Definition 2 .

Consider now the third output, defining the set $f^{I}$. From the definition of $\mathcal{R}_{f}$, it follows that for each function in $\mathcal{F}\left(\mathcal{R}_{f}\right), f^{I}$ can get the value 1 only on the points of $I$, while is always 0 outside $I$. Moreover, on each point of $I$ there is always the possibility of choosing between a 1 and a don't care value, so that $\emptyset \subseteq f^{I} \subseteq I$.

To complete the proof we must show that for any function compatible with $\mathcal{R}_{f}$, the P-circuit $P(f)$ constructed using the tuple $\left(f^{=}, f^{\neq}, f^{I}\right)$ is a P-circuit for $f$, i.e., $f^{o n} \subseteq P(f) \subseteq f^{o n} \cup f^{d c}$. This follows from the way the relation is defined on the points of $I$. Recall that each point $x$ in the intersection $I$ corresponds to a pair of points of the original space $\{0,1\}^{n}$, one projected onto $x_{i}=p$ and the other onto $x_{i} \neq p$, where $f$ is equal to 1 or to a don't care. $\mathcal{R}_{f}$ is such that

- if $x \in I$ corresponds to two minterms in $f^{o n}$, then in all possible outputs $\mathcal{R}_{f}(x)$, we have that either the third output $f^{I}$ is set to 1 , thus $x$ is covered by the SOP $S\left(f^{I}\right)$, or both the first and the second output $f^{=}$and $f^{\neq}$are set to 1 , and $x$ is covered by the corresponding SOPs in both projection sets;

- for each point $x \in I$ corresponding to a minterm of one cofactor and to a don't care of the other cofactor, we have one of two cases: either we set to $1 f=$ (if $\left.x \in f^{o n}\right|_{x_{i}=p}$ ) or $f^{\neq}$(if $\left.x \in f^{o n}\right|_{x_{i} \neq p}$ ), and therefore $x$ is covered by $S\left(f^{=}\right)$or $S\left(f^{\neq}\right)$, or the third output is set to 1 and therefore $x$ is covered by $S\left(f^{I}\right)$;

- if $x \in I$ corresponds to a pair of don't cares of $f$, then $R_{f}$ sets it to a don't care in all sets, so that it might be covered by $S\left(f^{I}\right)$, or $S\left(f^{=}\right)$, or $S\left(f^{\neq}\right)$, or none of them.

We finally prove that under the hypothesis that the projection function $p$ is the constant function $p=0$, an optimal solution of $\mathcal{R}_{f}$ provides an optimal P-circuit for $f$.

Corollary 1: For $p=0$, the optimal solution of the Boolean relation $\mathcal{R}_{f}$, according to the cost function $\mu$ chosen to evaluate the circuits $P(f)$, defines an optimal P-circuit $P^{*}(f)$ for the function $f$ with respect to the same cost function $\mu$.

Proof: The thesis immediately follows from Theorem 1 , as any three-output function compatible with $\mathcal{R}_{f}$ defines a possible P-circuit implementation for $f$. Indeed, with this choice of $p$ we have that $P(f)=\bar{x}_{i} S\left(f^{=}\right)+$ $x_{i} S\left(f^{\neq}\right)+S\left(f^{I}\right)$, and its cost, under any given cost metric $\mu$, is determined by the cost under $\mu$ of the SOP representations of $f^{=}, f^{\neq}$and $f^{I}$.

From Theorem 1 and Corollary 1 we directly have the following corollary.
Corollary 2: For $p=0$, the circuit computed by the algorithm in Figure 8, optimized according to a given cost function $\mu$, is an optimal P-circuit $P^{*}(f)$ for the function $f$ with respect to the same cost function $\mu$.

Finally, we can prove that, when $p=0$, the complexity of modeling P-circuit synthesis using Boolean relations is super-linear in the truth table representation of the input function $f$.

Theorem 2: Given a truth table representation of a completely specified Boolean function $f$ defined over $n$ binary variables, the construction of the Boolean relation $\mathcal{R}_{f}$, for $p=0$, takes time and space upper-bounded by $O(k \log k)$, when the input dimension is $k=2^{n}$.

Proof: The truth table representation of a Boolean function $f:\{0,1\}^{n} \rightarrow\{0,1\}$ consists in $k=2^{n}$ bits. Suppose to generate all the $k=2^{n}$ Boolean points in $\{0,1\}^{n}$ each containing $n$ bits. This operation costs $O\left(n 2^{n}\right)=O(k \log k)$. The computational time for deriving the projections $\left.f\right|_{x_{i}=0}$ and $\left.f\right|_{x_{i} \neq 0}$ is then linear in $k$, since we have to derive the points in the on-set of $f$ that have $x_{i}=0$ and $x_{i}=1$, respectively.

The computation of the intersection $I=\left.\left.f\right|_{x_{i}=0} \cap f\right|_{x_{i} \neq 0}$ can be performed in time $O(k \log k)$ with the following strategy: 1) we sort the points in $\left.f\right|_{x_{i}=0}$ and $\left.f\right|_{x_{i} \neq 0}$ using a linear time sorting algorithm (e.g., radix sort [9]) in time $O\left((n-1) 2^{n-1}\right)$ (recall that $\left.f\right|_{x_{i}=0}$ and $\left.f\right|_{x_{i} \neq 0}$ are sets containing vectors of $n-1$ bits); 2) we can perform the intersection of the two sorted sets in time $O\left((n-1) 2^{n-1}\right)$ using a strategy similar to the merge procedure in the Mergesort [9]. Similarly, the computation of $\left.f\right|_{x_{i}=0} \backslash I$ and $\left.f\right|_{x_{i} \neq 0} \backslash I$ costs $O\left((n-1) 2^{n-1}\right)$, since $\left.f\right|_{x_{i}=0},\left.f\right|_{x_{i} \neq 0}$ and $I$ are sorted.

Given $\left.f\right|_{x_{i}=0} \backslash I,\left.f\right|_{x_{i} \neq 0} \backslash I$ and $I$, the construction of the relation $R_{f}$ is then $O\left(n 2^{n}\right)$. In fact, as shown by the algorithm in Figure 6, to each cube in $\left.f\right|_{x_{i}=0} \backslash I,\left.f\right|_{x_{i} \neq 0} \backslash I$, and $I$ corresponds an entry in the Boolean relation; since we have $O\left(2^{n}\right)$ cubes of $O(n)$ variables, this phase costs $O\left(n 2^{n}\right)$. The overall procedure takes time and space upperbounded by $O\left(n 2^{n}\right)=O(k \log k)$.

Note that we can use OBDDs for computing $\left.f\right|_{x_{i}=0} \backslash I$, $\left.f\right|_{x_{i} \neq 0} \backslash I$, and $I$. This will give a computational complexity that depends on the sizes of the OBDDs.

We have the same result for incompletely specified functions with a similar proof.

\subsubsection{General $\mathbf{p}$}

Consider now the case where we project with respect to a general $p$. Now if we want to construct an optimal P-circuit we must take also into account the SOP $S(p)$ for $p$. For this purpose, we reformulate the problem with a four output relation $\mathcal{R}_{f}^{p}$ where the output set describes all the possible tuples of functions $f^{=}, f^{\neq}, f^{I}$, and $p$. Thus we must double the cases enumerated for the relation $\mathcal{R}_{f}$. In fact, each case must consider the points that are also in $p$ and the points that do not intersect $p$ : the former points must have a 1 as a final output, while the latter ones must have a 0 . For example, if we consider a completely specified function $f$, we have the following relation $\mathcal{R}_{f}^{p}$ : 


\begin{tabular}{|l|c|}
\hline$x_{1} \ldots x_{i-1} x_{i+1} \ldots x_{n}$ & $\mathcal{R}_{f}^{p}$ \\
\hline points in $\left(\left.f\right|_{x_{i}=p} \backslash I\right) \cap p$ & $\{1001\}$ \\
points in $\left(\left.f\right|_{x_{i}=p} \backslash I\right) \backslash p$ & $\{1000\}$ \\
points in $\left(\left.f\right|_{x_{i} \neq p} \backslash I\right) \cap p$ & $\{0101\}$ \\
points in $\left(\left.f\right|_{x_{i} \neq p} \backslash I\right) \backslash p$ & $\{0100\}$ \\
points in $I \cap p$ & $\{--11,11-1\}$ \\
points in $I \backslash p$ & $\{--10,11-0\}$ \\
points in $\left(\{0,1\}^{n-1} \backslash\left(\left.\left.f\right|_{x_{i}=p} \cup f\right|_{x_{i} \neq p} \cup I\right)\right) \cap p$ & $\{0001\}$ \\
all other points & $\{0000\}$ \\
\hline
\end{tabular}

Theorem 1 and Corollary 1 can then be easily generalized to the relation $\mathcal{R}_{f}^{p}$.

\subsection{Multioutput Functions}

In this section we discuss briefly how to handle a multioutput function $f:\{0,1\}^{n} \rightarrow\{0,1,-\}^{m}$.

There are two possible approaches. The first one consists simply in minimizing each output independently from the others, thus solving $m$ P-circuit minimization problems: we construct the $m$ Boolean relations related to the $m$ outputs of $f$, and find an optimal solution for each of them, independently.

The other possibility is to handle the $m$ outputs all together, defining a unique Boolean relation $\mathcal{R}_{f}$ with input set $\{0,1\}^{n-1}$, and output set $\{0,1\}^{3 m}$, featuring three outputs of $\mathcal{R}_{f}$ for each output of $f$. Thus, the problem of minimizing $f$ in P-circuit form is reduced to the problem of finding an optimal implementation of $\mathcal{R}_{f}$, according to the chosen cost metric.

However, this second approach presents some difficulties. In fact, a solution of the relation $\mathcal{R}_{f}$ defines correctly a Pcircuit for the function $f$ only if $\mathcal{R}_{f}$ is represented either in truth table form, or in an algebraic form whose products define a partition of $\{0,1\}^{n-1}$, i.e., the corresponding cubes are disjoint. In fact, when a minterm is represented by two different products, the solution of the relation would choose one of the possible associated outputs.

For example, consider a two-output function. In order to correctly partition the input points for the relation, we have to consider points that are in the first output and not in the second, then points that are in the first and in the second, and so on, including all the possible combinations of cases (e.g., points in $f^{=}, f^{\neq}$, etc.), as done for $p$ in the definition of the relation $\mathcal{R}_{f}^{p}$. Therefore, the construction of the relation $\mathcal{R}_{f}$ could become exponential in time and space. Table 1 shows the relation $\mathcal{R}_{f^{1}, f^{2}}:\{0,1\}^{n-1} \rightarrow$ $\{0,1\}^{6}$ for a completely specified multiple output function $f$ with two outputs: $f^{1}$ and $f^{2}$, where $I^{1}=\left.f^{1}\right|_{x_{i}=p} \cap$ $\left.f^{1}\right|_{x_{i} \neq p}$ and $I^{2}=\left.\left.f^{2}\right|_{x_{i}=p} \cap f^{2}\right|_{x_{i} \neq p}$.

Theorem 3: Given a SOP representation of a multioutput completely specified Boolean function $f:\{0,1\}^{n} \rightarrow$ $\{0,1,-\}^{m}$, the construction of the Boolean relation $\mathcal{R}_{f}$ takes time upper-bounded by $O\left(m n 2^{n} 2^{m}\right)$.

Proof: For each output $f^{j}$ (with $1 \leq j \leq m$ ) we construct $\left.f^{j}\right|_{x_{i}=0} \backslash I^{j},\left.f^{j}\right|_{x_{i} \neq 0} \backslash I^{j}$, and $I^{j}$. From the proof of Theorem 2 we have that the complexity of this phase is $O\left(m n 2^{n}\right)$. Then, we have to construct the relation's output set. Each minterm of the function $f$ may belong to the on-set of each output. Thus, we have to perform all the possible intersections of the $m$ outputs. For this purpose, we have to compute all the possible subsets of the set $\left\{f^{1}, f^{2}, \ldots, f^{m}\right\}$, whose number is $2^{m}$. Each intersection involves $O(m)$ outputs and each intersection between a couple of outputs costs $O\left(n 2^{n}\right)$. The overall cost of each intersection is then $O\left(m n 2^{n}\right)$ in time. Since we have to compute $2^{m}$ subsets, i.e., we have to perform $2^{m}$ intersections costing each $O\left(m n 2^{n}\right)$, the intersection phase costs $O\left(m n 2^{n} 2^{m}\right)$. The overall cost is then upper-bounded by $O\left(m n 2^{n} 2^{m}\right)$.

For this reason we chose to consider the outputs separately.

Note that if the input is a truth table, i.e., has dimension $m 2^{n}$, the costs $O\left(m n 2^{n} 2^{m}\right)$ of the construction is superlinear in $n$ but it is still exponential in $m$.

Moreover, the following example illustrates another related issue, due to how output cubes are interpreted by the Boolean relation minimizer BREL [2], which we use for our experiments. Consider the relation

$$
\begin{array}{cc}
0- & \{1010,0001\} \\
-1 & \{0100\} \\
10 & \{----\}
\end{array}
$$

where the minterm 01 is represented by both products $0-$ and -1 . If we minimize $\mathcal{R}$, we get the following function

$$
\begin{array}{ll}
-1 & 0100 \\
-0 & 0001
\end{array}
$$

which is compatible with $\mathcal{R}$, and therefore represents a correct solution for the relation.

However, if the relation $\mathcal{R}$ is a PLA specification of a four-output function $f$, according to the semantic of a classical SOP miminizer as ESPRESSO [12], output 1 prevails on output 0 in case of intersections among products. In this interpretation, the found solution does not represent correctly a SOP expression for $f$, since for the input 01 we get 0100 , while the correct output should be 1110 or 0101 . Observe that to obtain an interpretation á la ESPRESSO, we should change the starting relation defining the input mintems separately as follows:

$\begin{array}{cc}00 & \{1010,0001\} \\ 01 & \{1110,0101\} \\ 11 & \{0100\} \\ 10 & \{----\}\end{array}$

\section{EXPERIMENTAL Results}

In this section we report the experimental results for the P-circuits derived by the synthesis of Boolean relations.

We evaluate and report the area, delay, and power dissipation for the cases where $p$ is a constant (i.e., $p=0$ ) and $p$ is a variable (i.e., $p=V A R$ ).

The algorithms have been implemented in $\mathrm{C}$, using the CUDD library for OBDDs to represent Boolean functions, and BREL [2] for the synthesis of Boolean relations since, as far as we know, it finds better solutions in shorter runtime than the previous methods. 
TABLE 1

Boolean relation $\mathcal{R}_{f^{1}, f^{2}}$ for a completely specified multiple output function $f$ with two outputs: $f^{1}$ and $f^{2}$.

\begin{tabular}{|c|c|}
\hline$x_{1} \ldots x_{i-1} x_{i+1} \ldots x_{n}$ & $\mathcal{R}_{f^{1}, f^{2}}$ \\
\hline 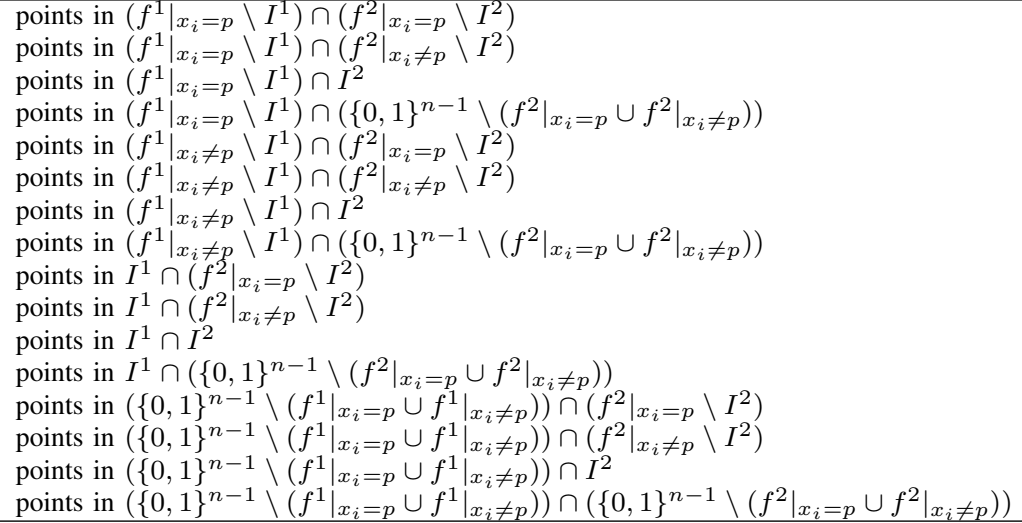 & $\begin{array}{c}\{100100\} \\
\{100010\} \\
\{100--1,10011-\} \\
\{100000\} \\
\{010100\} \\
\{010010\} \\
\{010--1,01011-\} \\
\{010000\} \\
\{--1100,11-100\} \\
\{--1010,11-010\} \\
\{--1--1,--111-, 11---1,11-11-\} \\
\{--1000,11-000\} \\
\{000100\} \\
\{000010\} \\
\{000-1,00011-\} \\
\{000000\}\end{array}$ \\
\hline
\end{tabular}

TABLE 2

Synthesis time (in seconds), mapped area and delay of P-circuits, S-circuits, and SOP forms (case $p=0$ ).

\begin{tabular}{|c|c|c|c|c|c|c|c|c|c|c|c|c|c|c|c|}
\hline \multirow{2}{*}{$\begin{array}{l}\text { Benchmark } \\
\text { (in/out) }\end{array}$} & \multicolumn{3}{|c|}{ P-circuit $\mu_{L}$} & \multicolumn{3}{|c|}{ P-circuit $\mu_{B D D}$} & \multicolumn{3}{|c|}{ P-circuit [6] } & \multicolumn{3}{|c|}{ S-circuit } & \multicolumn{3}{|c|}{ SOP } \\
\hline & Time & Area & Delay & Time & Area & Delay & Time & Area & Delay & Time & Area & Delay & Time & Area & Delay \\
\hline b10 $(15 / 11)$ & 9.75 & 850 & 39.1 & 0.18 & 938 & 38.7 & 0.05 & 1067 & 42.0 & 0.07 & 1113 & 47.5 & 0.01 & 881 & 45.2 \\
\hline b2 $(16 / 17)$ & 103.82 & 3591 & 68.2 & 1.68 & 3757 & 69.9 & 0.66 & 4479 & 82.8 & 10.19 & 6281 & 115.4 & 0.01 & 3957 & 73.0 \\
\hline b3 $(32 / 20)$ & 168.10 & 949 & 28.4 & 0.13 & 1002 & 29.5 & 0.93 & 1134 & 39.0 & 0.82 & 1284 & 40.1 & 0.03 & 1095 & 44.7 \\
\hline $\operatorname{ex1010}(10 / 10)$ & 50.65 & 2923 & 81.0 & 0.61 & 4170 & 96.1 & 0.39 & 4271 & 102.3 & 0.58 & 4818 & 113.5 & 0.31 & 4254 & 95.1 \\
\hline ex4 (128/28) & 18.51 & 800 & 21.8 & 1.15 & 1099 & 27.3 & 0.22 & 806 & 25.0 & 0.40 & 1520 & 29.6 & 0.10 & 801 & 25.0 \\
\hline exam $(10 / 10)$ & 5.78 & 408 & 30.0 & 0.14 & 475 & 28.8 & 0.08 & 655 & 29.0 & 0.12 & 617 & 32.7 & 0.07 & 526 & 31.8 \\
\hline $\operatorname{exep}(30 / 63)$ & 1.89 & 1305 & 30.5 & 0.03 & 1315 & 28.5 & 0.04 & 1347 & 31.6 & 0.07 & 1321 & 30.5 & 0.01 & 1275 & 36.1 \\
\hline gary $(15 / 11)$ & 11.98 & 1000 & 41.1 & 0.19 & 1096 & 44.9 & 0.04 & 1189 & 44.9 & 0.04 & 1304 & 50.3 & 0.01 & 1030 & 53.9 \\
\hline ibm $(48 / 17)$ & 13.33 & 741 & 30.1 & 0.58 & 1117 & 35.4 & 0.04 & 741 & 30.1 & 0.08 & 1368 & 42.9 & 0.01 & 700 & 29.0 \\
\hline in3 $(35 / 29)$ & 4.32 & 929 & 34.5 & 0.15 & 968 & 36.2 & 0.06 & 1157 & 35.8 & 0.04 & 1153 & 38.6 & 0.01 & 1111 & 34.1 \\
\hline in $4(32 / 20)$ & 167.37 & 1002 & 28.5 & 0.13 & 1055 & 29.6 & 0.77 & 1192 & 38.0 & 0.56 & 1331 & 40.2 & 0.02 & 1077 & 44.8 \\
\hline jbp $(36 / 57)$ & 4.42 & 1059 & 33.8 & 0.16 & 1310 & 35.4 & 0.05 & 1193 & 38.6 & 1.38 & 1690 & 40.0 & 0.02 & 1115 & 35.9 \\
\hline m4 (8/16) & 7.20 & 783 & 36.6 & 0.01 & 849 & 39.5 & 0.02 & 2274 & 71.4 & 0.02 & 2766 & 85.3 & 0.03 & 1778 & 54.2 \\
\hline mainpla $(27 / 54)$ & 338.55 & 15118 & 205.1 & 6.58 & 15610 & 217.5 & 12.17 & 26531 & 345.9 & 337.04 & 24421 & 335.2 & 0.08 & 25529 & 371.0 \\
\hline $\max 1024(10 / 6)$ & 33.96 & 1408 & 47.3 & 0.02 & 1554 & 58.4 & 0.08 & 2755 & 70.6 & 0.07 & 2534 & 75.2 & 0.14 & 1690 & 53.7 \\
\hline $\operatorname{misg}(56 / 23)$ & 1.38 & 153 & 18.2 & 0.04 & 167 & 18.2 & 0.01 & 153 & 18.2 & 0.04 & 348 & 23.0 & 0.01 & 152 & 18.2 \\
\hline mish $(94 / 43)$ & 0.54 & 168 & 10.0 & 0.07 & 207 & 10.0 & 0.09 & 168 & 10.0 & 0.29 & 427 & 13.9 & 0.01 & 168 & 9.4 \\
\hline misj $(35 / 14)$ & 0.52 & 81 & 9.7 & 0.03 & 125 & 9.1 & 0.01 & 81 & 8.9 & 0.01 & 184 & 13.9 & 0.01 & 81 & 8.9 \\
\hline pdc $(16 / 40)$ & 1.83 & 683 & 26.8 & 0.03 & 722 & 26.4 & 0.40 & 1555 & 46.8 & 0.53 & 826 & 34.7 & 0.44 & 1633 & 44.0 \\
\hline prom2 $(9 / 21)$ & 18.61 & 4612 & 115.1 & 0.01 & 4905 & 117.0 & 0.42 & 7462 & 171.4 & 0.24 & 7226 & 175.1 & 0.18 & 6775 & 185.1 \\
\hline spla $(16 / 46)$ & 3.24 & 1826 & 50.6 & 0.01 & 1935 & 50.7 & 0.17 & 2284 & 59.9 & 0.15 & 2239 & 53.8 & 0.21 & 2470 & 68.6 \\
\hline ts10 $(22 / 16)$ & 43.40 & 942 & 36.5 & 0.50 & 1410 & 44.9 & 0.17 & 942 & 36.5 & 0.16 & 1806 & 66.1 & 0.01 & 901 & 35.2 \\
\hline vg2 $(25 / 8)$ & 5.15 & 577 & 22.8 & 0.06 & 632 & 23.9 & 0.01 & 603 & 23.2 & 0.01 & 546 & 25.6 & 0.01 & 341 & 18.6 \\
\hline x6dn $(39 / 5)$ & 10.64 & 760 & 30.8 & 0.26 & 788 & 31.6 & 0.01 & 874 & 35.9 & 0.01 & 885 & 35.1 & 0.01 & 762 & 31.2 \\
\hline x7dn $(66 / 15)$ & 64.15 & 2378 & 51.1 & 0.01 & 2478 & 51.8 & 0.51 & 2501 & 63.8 & 0.54 & 2371 & 49.9 & 0.12 & 1544 & 46.1 \\
\hline x9dn $(27 / 7)$ & 3.30 & 412 & 24.1 & 0.04 & 425 & 24.5 & 0.02 & 412 & 24.1 & 0.02 & 530 & 27.9 & 0.01 & 384 & 23.0 \\
\hline xparc $(41 / 73)$ & 84.08 & 9175 & 121.2 & 0.32 & 9280 & 126.9 & 0.51 & 13478 & 187.3 & 0.53 & 13357 & 180.4 & 0.14 & 12678 & 168.8 \\
\hline
\end{tabular}

The experiments have been run on a Linux Intel Core i7, $3.40 \mathrm{GHz} \mathrm{CPU}$ with $8 \mathrm{~GB}$ of main memory. The benchmarks are taken from LGSynth93 [15]. We report in the following a significant subset of the functions as representative indicators of our experiments.

\subsection{Area minimization}

To show the gain in area of P-circuits derived using Boolean relations, we compare our results with the algorithm based on don't cares reported in [6].

To highlight the importance of projecting only part of the intersection, we report the results regarding circuits decomposed with standard Shannon decomposition (therefore without any intersection), called here S-circuits. We compare our results also with plain SOP forms, whose
TABLE 3

Average gain of $\mathrm{P}$-circuits based on Boolean relations (case $p=0$ ).

\begin{tabular}{|c|ccl||ccc|}
\hline & \multicolumn{3}{|c|}{ P-circuit $\mu_{L}$} & \multicolumn{3}{c|}{ P-circuit $\mu_{B D D}$} \\
\cline { 2 - 7 } Average gain & Time & Area & Delay & Time & Area & Delay \\
\hline w.r.t. S-circuit & $-383 \%$ & $37 \%$ & $29 \%$ & $95 \%$ & $30 \%$ & $25 \%$ \\
w.r.t. P-circuit [6] & $-4214 \%$ & $33 \%$ & $24 \%$ & $56 \%$ & $25 \%$ & $20 \%$ \\
w.r.t. SOP & $-39412 \%$ & $26 \%$ & $19 \%$ & $-304 \%$ & $18 \%$ & $14 \%$ \\
\hline
\end{tabular}

minimization does not even take into account the critical variable $x_{i}$.

The SOP circuits and the projections of the SOP components of the S-circuits have been synthesized using ESPRESSO in the heuristic mode. To evaluate the obtained circuits, we ran them using the SIS system with the MCNC library for technology mapping and the SIS command map 
TABLE 4

Synthesis time (in seconds), mapped area and delay of P-circuits (case $p=V A R$ ).

\begin{tabular}{|c|c|c|c|c|c|c|c|c|c|c|c|c|c|c|c|}
\hline \multirow{2}{*}{$\begin{array}{l}\text { Benchmark } \\
\text { (in/out) }\end{array}$} & \multicolumn{3}{|c|}{ P-circuit $\mu_{L}$} & \multicolumn{3}{|c|}{ P-circuit $\mu_{B D D}$} & \multicolumn{3}{|c|}{ P-circuit [6] } & \multicolumn{3}{|c|}{ S-circuit } & \multicolumn{3}{|c|}{ SOP } \\
\hline & Time & Area & Delay & Time & Area & Delay & Time & Area & Delay & Time & Area & Delay & Time & Area & Delay \\
\hline b10 $(15 / 11)$ & 9,78 & 829 & 39,70 & 0,15 & 914 & 38,50 & 0,05 & 1067 & 42,0 & 0,07 & 1113 & 47,5 & 0,00 & 881 & 45,2 \\
\hline b2 $(16 / 17)$ & 128,78 & 4864 & 84,80 & 1,74 & 5096 & 97,20 & 0,66 & 4479 & 82,8 & 10,19 & 6281 & 115,4 & 0,01 & 3957 & 73,0 \\
\hline b3 $(32 / 20)$ & 166,63 & 963 & 32,30 & 0,12 & 1015 & 34,40 & 0,93 & 1134 & 39,0 & 0,82 & 1284 & 40,1 & 0,03 & 1095 & 44,7 \\
\hline $\operatorname{ex1010}(10 / 10)$ & 48,37 & 2977 & 76,00 & 0,58 & 4083 & 93,50 & 0,39 & 4271 & 102,3 & 0,58 & 4818 & 113,5 & 0,31 & 4254 & 95,1 \\
\hline ex4 $(128 / 28)$ & 18,73 & 872 & 24,00 & 1,17 & 1210 & 30,20 & 0,22 & 806 & 25,0 & 0,40 & 1520 & 29,6 & 0,10 & 801 & 25,0 \\
\hline exam $(10 / 10)$ & 6,15 & 479 & 28,20 & 0,12 & 542 & 31,70 & 0,08 & 655 & 29,0 & 0,12 & 617 & 32,7 & 0,07 & 526 & 31,8 \\
\hline $\operatorname{exep}(30 / 63)$ & 1,80 & 1329 & 45,80 & 0,04 & 1352 & 45,90 & 0,04 & 1347 & 31,6 & 0,07 & 1321 & 30,5 & 0,01 & 1275 & 36,1 \\
\hline gary $(15 / 11)$ & 12,12 & 960 & 41,10 & 0,16 & 1064 & 40,20 & 0,04 & 1189 & 44,9 & 0,04 & 1304 & 50,3 & 0,00 & 1030 & 53,9 \\
\hline ibm (48/17) & 13,64 & 780 & 31,40 & 0,63 & 1145 & 35,50 & 0,04 & 741 & 30,1 & 0,08 & 1368 & 42,9 & 0,00 & 700 & 29,0 \\
\hline in3 $(35 / 29)$ & 4,43 & 942 & 44,60 & 0,13 & 997 & 45,60 & 0,06 & 1157 & 35,8 & 0,04 & 1153 & 38,6 & 0,00 & 1111 & 34,1 \\
\hline in4 $(32 / 20)$ & 166,34 & 1017 & 32,40 & 0,12 & 1069 & 34,50 & 0,77 & 1192 & 38,0 & 0,56 & 1331 & 40,2 & 0,02 & 1077 & 44,8 \\
\hline jbp $(36 / 57)$ & 4,15 & 1165 & 41,40 & 0,14 & 1438 & 55,40 & 0,05 & 1193 & 38,6 & 1,38 & 1690 & 40,0 & 0,02 & 1115 & 35,9 \\
\hline m4 (8/16) & 6,91 & 809 & 33,90 & 0,03 & 876 & 36,30 & 0,02 & 2274 & 71,4 & 0,02 & 2766 & 85,3 & 0,03 & 1778 & 54,2 \\
\hline mainpla $(27 / 54)$ & 314,85 & 14355 & 225,40 & 5,51 & 14801 & 247,70 & 12,17 & 26531 & 345,9 & 337,04 & 24421 & 335,2 & 0,08 & 25529 & 371,0 \\
\hline $\operatorname{max1024}(10 / 6)$ & 37,79 & 1396 & 57,10 & 0,01 & 1563 & 60,90 & 0,08 & 2755 & 70,6 & 0,07 & 2534 & 75,2 & 0,14 & 1690 & 53,7 \\
\hline $\operatorname{misg}(56 / 23)$ & 1,34 & 159 & 18,20 & 0,04 & 173 & 18,20 & 0,00 & 153 & 18,2 & 0,04 & 348 & 23,0 & 0,00 & 152 & 18,2 \\
\hline mish $(94 / 43)$ & 0,53 & 178 & 10,80 & 0,05 & 218 & 15,10 & 0,09 & 168 & 10,0 & 0,29 & 427 & 13,9 & 0,00 & 168 & 9,4 \\
\hline misj (35/14) & 0,51 & 87 & 9,70 & 0,01 & 130 & 13,30 & 0,00 & 81 & 8,9 & 0,00 & 184 & 13,9 & 0,00 & 81 & 8,9 \\
\hline pdc $(16 / 40)$ & 0,82 & 670 & 36,00 & 0,02 & 690 & 35,50 & 0,40 & 1555 & 46,8 & 0,53 & 826 & 34,7 & 0,44 & 1633 & 44,0 \\
\hline prom2 $(9 / 21)$ & 18,26 & 4694 & 115,80 & 0,00 & 5029 & 120,40 & 0,42 & 7462 & 171,4 & 0,24 & 7226 & 175,1 & 0,18 & 6775 & 185,1 \\
\hline spla $(16 / 46)$ & 2,84 & 1766 & 50,70 & 0,00 & 1860 & 50,00 & 0,17 & 2284 & 59,9 & 0,15 & 2239 & 53,8 & 0,21 & 2470 & 68,6 \\
\hline ts10 $(22 / 16)$ & 43,63 & 1046 & 38,80 & 0,47 & 1503 & 46,40 & 0,17 & 942 & 36,5 & 0,16 & 1806 & 66,1 & 0,00 & 901 & 35,2 \\
\hline vg2 $(25 / 8)$ & 4,34 & 541 & 21,30 & 0,03 & 625 & 21,90 & 0,00 & 603 & 23,2 & 0,01 & 546 & 25,6 & 0,00 & 341 & 18,6 \\
\hline x6dn $(39 / 5)$ & 10,19 & 763 & 31,60 & 0,24 & 792 & 32,00 & 0,01 & 874 & 35,9 & 0,00 & 885 & 35,1 & 0,00 & 762 & 31,2 \\
\hline x7dn $(66 / 15)$ & 58,77 & 2376 & 56,00 & 0,00 & 2490 & 51,80 & 0,51 & 2501 & 63,8 & 0,54 & 2371 & 49,9 & 0,12 & 1544 & 46,1 \\
\hline x9dn $(27 / 7)$ & 3,32 & 450 & 25,80 & 0,04 & 400 & 23,80 & 0,02 & 412 & 24,1 & 0,02 & 530 & 27,9 & 0,00 & 384 & 23,0 \\
\hline xparc $(41 / 73)$ & 68,68 & 9165 & 115,80 & 0,25 & 9212 & 120,50 & 0,51 & 13478 & 187,3 & 0,53 & 13357 & 180,4 & 0,14 & 12678 & 168,8 \\
\hline
\end{tabular}

TABLE 5

Average gain of $\mathrm{P}$-circuits based on Boolean relations (case $p=V A R$ ).

\begin{tabular}{|c|ccc||ccc|}
\hline & \multicolumn{3}{|c||}{ P-circuit $\mu_{L}$} & \multicolumn{3}{c|}{ P-circuit $\mu_{B D D}$} \\
\cline { 2 - 7 } Average gain & Time & Area & Delay & Time & Area & Delay \\
\hline w.r.t. S-circuit & $-395 \%$ & $33 \%$ & $20 \%$ & $95 \%$ & $26 \%$ & $15 \%$ \\
w.r.t. P-circuit [6] & $-4326 \%$ & $28 \%$ & $14 \%$ & $59 \%$ & $21 \%$ & $8 \%$ \\
w.r.t. SOP & $-40440 \%$ & $21 \%$ & $8 \%$ & $-277 \%$ & $13 \%$ & $2 \%$ \\
\hline
\end{tabular}

$-\mathrm{W}-\mathrm{f} 3-\mathrm{s}$.

In Table 2 we compare synthesis time (in seconds), mapped area and delay of P-circuits, S-circuits, and SOP forms for a significant subset of the benchmarks (i.e. a subset that has on average the same characteristics of the entire set of benchmarks), considering $p=0$ and $x_{i}=x_{0}$. The first column reports the name of the benchmarks and the number of their inputs and outputs. The following ones report, by groups of three, the synthesis times in seconds, and the areas and delays estimated by SIS. The first two groups, labeled P-circuit $\mu_{L}$ and $\mathrm{P}$-circuit $\mu_{B D D}$, refer to P-circuits synthesized with the new algorithm based on Boolean relations with cost function $\mu_{L}$ that minimizes the number of literals, and $\mu_{B D D}$ that minimizes the size of the BDDs used for representing the relations. The next group refers to P-circuits synthesized with the minimization strategy proposed in [6]. The last two groups provide the results for S-circuits and SOP forms. For each benchmark we underline in bold the circuit that exhibits better area results.

The results show that modeling the P-circuit minimization problem using Boolean relations pays significantly. In fact, P-circuits synthesized with Boolean relations (P-circuit $\mu_{L}$ and P-circuit $\left.\mu_{B D D}\right)$ turned out to be more compact

\section{TABLE 6}

$p=0$ vs. $p=V A R$ : percentage of benchmarks where the choice $p=0$ exhibits better results than $p=V A R$.

\begin{tabular}{|ccc||ccc|}
\hline \multicolumn{3}{|c||}{ P-circuit $\mu_{L}$} & \multicolumn{3}{c|}{ P-circuit $\mu_{B D D}$} \\
\hline Time & Area & Delay & Time & Area & Delay \\
\hline $45 \%$ & $78 \%$ & $78 \%$ & $76 \%$ & $77 \%$ & $79 \%$ \\
\hline
\end{tabular}

than the corresponding P-circuits proposed in [6] in about 92\% and $78 \%$ of our experiments, respectively.

The area gain of P-circuits synthesized with Boolean relations and cost function $\mu_{L}\left(\mu_{B D D}\right)$ is $33 \%(25 \%)$ in the average w.r.t. P-circuits in [6]. Several benchmarks show gains above the $60 \%$ (see, for example $m 4$ with $66 \%$ of gain for $\mu_{L}$ and $63 \%$ for $\mu_{B D D}$ ).

The area gain w.r.t. SOP forms is also very interesting, since P-circuits are designed for dealing with critical variables and thus area is of secondary importance. The Pcircuits synthesized with Boolean relations and cost function $\mu_{L}\left(\mu_{B D D}\right)$ are more compact than the corresponding SOP forms in about $72 \%$ (60\%) of our experiments, with an average gain in area of $26 \%(18 \%)$.

Comparing the performances of the two new algorithms with respect to the P-circuit algorithm proposed in [6], we notice how the cost function can be critical: $\mu_{L}$ can be quite time-expensive $(4214 \%$ penalty in computational time, on average, w.r.t. [6]), while $\mu_{B D D}$ is the best-performing algorithm (56\% gain in computational time, on average, w.r.t. [6]).

Moreover, the delay gain of P-circuits synthesized with Boolean relations and cost function $\mu_{L}\left(\mu_{B D D}\right)$ is $24 \%$ $(20 \%)$ in the average w.r.t. P-circuits in [6]. This is a good result, considering that we did not perform a synthesis to reduce explicitly the delay. It is reasonable to assume that 
TABLE 7

Power dissipation (in Watt) of P-circuits (case $p=0$ ), S-circuits, and SOP forms.

\begin{tabular}{|c|c|c|c|c|c|c|}
\hline Benchmark & in/out & P-circuit $\mu_{L}$ & P-circuit $\mu_{B D D}$ & P-circuit [6] & S-circuit & SOP \\
\hline b3 & $32 / 20$ & $5.04 \mathrm{E}-03$ & $\mathbf{1 . 1 8 E - 0 4}$ & $4.40 \mathrm{E}-03$ & $5.61 \mathrm{E}-03$ & $5.70 \mathrm{E}-03$ \\
bench1 & $9 / 9$ & $7.17 \mathrm{E}-03$ & $5.27 \mathrm{E}-03$ & $\mathbf{4 . 7 3 E - 0 3}$ & $5.64 \mathrm{E}-03$ & $7.31 \mathrm{E}-03$ \\
ex1010 & $10 / 10$ & $1.48 \mathrm{E}-02$ & $\mathbf{7 . 0 2 E}-03$ & $9.25 \mathrm{E}-03$ & $1.11 \mathrm{E}-02$ & $1.23 \mathrm{E}-02$ \\
ibm & $48 / 17$ & $\mathbf{3 . 4 9 E}-03$ & $1.69 \mathrm{E}-02$ & $3.52 \mathrm{E}-03$ & $7.21 \mathrm{E}-03$ & $3.91 \mathrm{E}-03$ \\
in3 & $35 / 29$ & $6.43 \mathrm{E}-03$ & $4.99 \mathrm{E}-03$ & $\mathbf{4 . 9 4 E - 0 3}$ & $6.46 \mathrm{E}-03$ & $5.21 \mathrm{E}-03$ \\
in4 & $32 / 20$ & $5.39 \mathrm{E}-03$ & $6.24 \mathrm{E}-03$ & $\mathbf{4 . 7 0 E - 0 3}$ & $5.80 \mathrm{E}-03$ & $5.82 \mathrm{E}-03$ \\
jbp & $36 / 57$ & $5.53 \mathrm{E}-03$ & $5.63 \mathrm{E}-03$ & $\mathbf{4 . 9 3 E - 0 3}$ & $1.15 \mathrm{E}-02$ & $6.08 \mathrm{E}-03$ \\
max1024 & $10 / 6$ & $7.91 \mathrm{E}-03$ & $7.89 \mathrm{E}-03$ & $\mathbf{7 . 1 0 E}-03$ & $7.46 \mathrm{E}-03$ & $9.81 \mathrm{E}-03$ \\
misg & $56 / 23$ & $3.29 \mathrm{E}-03$ & $8.30 \mathrm{E}-03$ & $1.51 \mathrm{E}-03$ & $1.04 \mathrm{E}-02$ & $\mathbf{1 . 4 4 E - 0 3}$ \\
misj & $35 / 14$ & $1.74 \mathrm{E}-03$ & $3.98 \mathrm{E}-03$ & $7.72 \mathrm{E}-04$ & $6.14 \mathrm{E}-03$ & $\mathbf{7 . 0 4 E - 0 4}$ \\
pdc & $16 / 40$ & $6.22 \mathrm{E}-03$ & $\mathbf{3 . 1 4 E - 0 3}$ & $6.26 \mathrm{E}-03$ & $6.43 \mathrm{E}-03$ & $7.70 \mathrm{E}-03$ \\
prom2 & $9 / 21$ & $1.43 \mathrm{E}-02$ & $\mathbf{6 . 3 9 E}-03$ & $1.31 \mathrm{E}-02$ & $1.40 \mathrm{E}-02$ & $1.08 \mathrm{E}-02$ \\
test4 & $8 / 30$ & $2.05 \mathrm{E}-02$ & $1.53 \mathrm{E}-02$ & $\mathbf{1 . 1 3 E}-02$ & $1.92 \mathrm{E}-02$ & $1.72 \mathrm{E}-02$ \\
ts10 & $22 / 16$ & $3.61 \mathrm{E}-03$ & $2.00 \mathrm{E}-02$ & $3.56 \mathrm{E}-03$ & $5.47 \mathrm{E}-03$ & $\mathbf{3 . 1 7 E - 0 3}$ \\
x7dn & $66 / 15$ & $1.20 \mathrm{E}-02$ & $\mathbf{5 . 0 8 E - 0 3}$ & $1.05 \mathrm{E}-02$ & $1.30 \mathrm{E}-02$ & $1.05 \mathrm{E}-02$ \\
xparc & $41 / 73$ & $8.20 \mathrm{E}-03$ & $1.39 \mathrm{E}-02$ & $\mathbf{3 . 8 0 E}-03$ & $8.71 \mathrm{E}-03$ & $3.99 \mathrm{E}-03$ \\
\hline
\end{tabular}

the delay gain is due to the reduction of the circuit area.

For a complete comparison of average gains see Table 3 .

Table 4 and Table 5 report the experimental results where $p$ is a variable (i.e., $p=x_{1}$ ), with $x_{i}=x_{0}$. Also in this case we can observe that the algorithm based on Boolean relations yields better results than the other minimization methods. In fact, P-circuits synthesized with Boolean relations (P-circuit $\mu_{L}$ and P-circuit $\mu_{B D D}$ ) turned out to be more compact than the corresponding P-circuits proposed in [6] in about $66 \%$ and $58 \%$ of our experiments, with an average gain, in area, of $28 \%$ and $21 \%$, respectively. Moreover, P-circuits synthesized with Boolean relations and cost function $\mu_{L}\left(\mu_{B D D}\right)$ are more compact than the corresponding SOP forms in about 55\% (47\%) of our experiments, with an average gain, in area, of $21 \%(13 \%)$.

As expected ([3], [4]), comparing the two sets of results (i.e. $p=0$ and $p=V A R$ ), we observe that the best choice for the projection function $p$ is often $p=0$. In Table 6 we report the percentages of benchmarks where the choice $p=0$ obtains better results w.r.t. the choice $p=V A R$, both for P-circuit $\mu_{L}$ and for P-circuit $\mu_{B D D}$.

In summary we can observe that the algorithm based on Boolean relations with cost function $\mu_{B D D}$ shows a good trade-off between area minimization and computational time.

\subsection{Power dissipation}

To evaluate power dissipation, the circuits were synthesized using the following equal-delay gates in a $180 \mathrm{~nm}$ CMOS technology: inverter, 2,3,4-input NAND, 2,3,4-input NOR and 2-input XOR gates. Since in CMOS technology at 180 $\mathrm{nm}$ the switching power is the predominant component of the overall power consumption, our model takes into account only this component.

The circuits were analyzed with two different average values of input transition rates, giving a high-density switching activity for only one known signal (in our case $x_{0}$ ) and a low-density activity for all other signals. The number of input logic transitions for the high-density switching activity signal is approximately one hundred times larger than the number of input logic transitions for the low-density switching activity signals.

The circuits have been simulated at transistor level using SPECTRE on a $1600 \mathrm{MHz}$ Pentium 4 workstation. The values of $i_{D D}$ and $i_{S S}$ currents were sampled and stored for post-processing. In all the experiments we considered for each benchmark decompositions with respect to the input variable with the highest switching frequency.

In Table 7 we compare power dissipation (in Watt) of P-circuits (case $p=0$ ), S-circuits, and SOP forms for a subset of the functions as representative indicators of our experiments. The first two columns report the name of the benchmarks and the number of their inputs and outputs. The following ones report the estimated power consumption. In particular, the third and fourth columns, labeled P-circuit $\mu_{L}$ and P-circuit $\mu_{B D D}$, respectively, refer to P-circuits synthesized with the new algorithm based on Boolean relations with cost function $\mu_{L}$ that minimizes the number of literals, and cost function $\mu_{B D D}$ that minimizes the size of the BDDs used for representing the relation. The next group refers to P-circuits synthesized with the minimization strategy proposed in [6]. The last two groups provide the results for S-circuits and SOP forms. For each benchmark we underline in bold the circuit that exhibits better results.

Table 8 summarizes the results obtained using Boolean relations. The power consumption of P-circuits synthesized with Boolean relations (P-circuit $\mu_{L}$ and P-circuit $\mu_{B D D}$ ) is lower than the power consumption of the corresponding Pcircuits proposed in [6] in about $13 \%$ of our experiments. This is a reasonable good result, since it is obtained by the comparison of the proposed approach (P-circuit with Boolean relation) against an approach (P-circuit [6]) that is itself an improvement with respect to other synthesis techniques (SOP and S-circuits). The percentage of improvement goes up if we compare P-circuits synthesized with Boolean relations against SOP and S-circuits.

In summary, if we compare Tables 3 and 8 , we observe that synthesis with cost function $\mu_{L}$ allows to obtain, on average, high gains in power and area/delay, at the expense 
TABLE 8

Comparison of power dissipation (case $p=0$ ).

\begin{tabular}{|c|c|c|}
\hline & P-circuit $\mu_{L}$ & P-circuit $\mu_{B D D}$ \\
\hline w.r.t. S-circuit & $65 \%$ & $61 \%$ \\
w.r.t. P-circuit [6] & $13 \%$ & $13 \%$ \\
w.r.t. SOP & $44 \%$ & $62 \%$ \\
\hline
\end{tabular}

of a significant increase in computational time. On the other hand, the P-circuits synthesized with the algorithm based on Boolean relations with cost function $\mu_{B D D}$ have a satisfactory gain in power, area and delay against a moderate increase of computational time. Therefore, the P-circuit synthesis based on the cost function $\mu_{B D D}$ is a good trade-off between power dissipation, computational time and area/delay minimization.

\section{Conclusion ANd Future Work}

In this paper we studied the problem of characterizing the complete flexibility of P-circuits and exploiting it. The previous algorithms did not necessarily find the best implementation, because they modeled the problem as minimizing an incompletely specified Boolean function. Here we showed that to explore all possible solutions one must set up and minimize a Boolean relation, because there are combinations of don't care conditions that cannot be expressed by cubes. The construction of the Boolean relation is super-linear in the input. In the experiments we report major improvements with respect to the previously published results.

Future work includes two main lines of investigation: evaluating the impact of more general cofactoring $p$ functions, and handling multiouput functions trading-off quality of results vs. scalability. About the former, currently we experimented mainly with $p=0$ and $p=V A R$, but we lack a systematic understanding of what $p$ are the best and how to choose them. About the latter, we performed the experiments only with single-output disjoint minimization of multioutput functions, because modeling with Boolean relations the complete flexibility of multioutput functions looks computationally too expensive, as shown in the related section. However, we would like to assess the quality gap between the two extreme algorithmic choices, and come up with Boolean relations that model only partially the sharing of the multi-output functions, to balance the objectives of more aggressive optimization vs. runtime. Moreover, how to integrate the proposed decomposition technique with other transformations of the Boolean network and its nodes requires more theoretical and experimental work to come up with a fine-tuned optimization script for multilevel synthesis. This is an interesting future direction.
[3] A. Bernasconi, V. Ciriani, G. Trucco, and T. Villa, "On Decomposing Boolean Functions via Extended Cofactoring," in Design Automation and Test in Europe (DATE), 2009, pp. 1464-1469.

[4] _- "Logic Synthesis by Signal-Driven Decomposition," in Advanced Techniques in Logic Synthesis, Optimizations and Applications, K. Gulati, Ed. Springer New York, 2011, pp. 9-29.

[5] - "Minimization of P-Circuits using Boolean Relations," in Design Automation and Test in Europe (DATE), 2013.

[6] A. Bernasconi, V. Ciriani, V. Liberali, G. Trucco, and T. Villa, "Synthesis of P-Circuits for Logic Restructuring," Integration, vol. 45, no. 3, pp. 282-293, 2012.

[7] J. C. Bioch, "The Complexity of Modular Decomposition of Boolean Functions," Discrete Applied Mathematics, vol. 149, no. 1-3, pp. 113, 2005.

[8] R. Brayton and F. Somenzi, "Boolean Relations and the Incomplete Specification of Logic Networks," in International Conference on Very Large Scale Integration - VLSI, August 1989.

[9] T. Cormen, C. Leiserson, R. Rivest, and C. Stein, Introduction to Algorithms, Third Edition. MIT Press, 2009.

[10] J. Cortadella, M. Kishinevsky, A. Kondratyev, L. Lavagno, E. Pastor, and A. Yakovlev, "Decomposition and Technology Mapping of Speed-Independent Circuits using Boolean Relations," IEEE Trans. on CAD of Integrated Circuits and Systems, vol. 18, no. 9, pp. 12211236, 1999.

[11] P. Kerntopf, "New Generalizations of Shannon Decomposition," in Int. Workshop on Applications of Reed-Muller Expansion in Circuit Design, 2001, pp. 109-118.

[12] P. McGeer, J. Sanghavi, R. Brayton, and A. Sangiovanni-Vincentelli, "ESPRESSO-SignATURE: A New Exact Minimizer for Logic Functions," IEEE Transactions on VLSI, vol. 1, no. 4, pp. 432-440, 1993.

[13] E. Sentovich, K. Singh, L. Lavagno, C. Moon, R. Murgai, A. Saldanha, H. Savoj, P. Stephan, R. Brayton, and A. SangiovanniVincentelli, "SIS: A System for Sequential Circuit Synthesis," Tech. Rep. No. UCB/ERL M92/41, Berkeley, CA, Tech. Rep., 1992.

[14] B. Sterin, A. Mishchenko, N. Een, and R. Brayton, "Logic Synthesis for Disjunctions of Boolean Functions," in IEEE/ACM 21 st International Workshop on Logic \& Synthesis (IWLS), 2012, pp. 73-77.

[15] S. Yang, "Logic Synthesis and Optimization Benchmarks User Guide Version 3.0," Microelectronic Center, User Guide, 1991.

\section{References}

[1] D. Bañeres, J. Cortadella, and M. Kishinevsky, "A Recursive Paradigm to Solve Boolean Relations," in Proc. ACM/IEEE Design Automation Conference, 2004, pp. 416-421.

[2] — "A Recursive Paradigm to Solve Boolean Relations," IEEE Transactions on Computers, vol. 58, no. 4, pp. 512-527, 2009. 